\title{
Nanomaterials-Based Photodynamic Therapy with Combined Treatment Improves Antitumor Efficacy Through Boosting Immunogenic Cell Death
}

\author{
Feiyang Jin' \\ Di Liu ${ }^{\prime}$ \\ Xiaoling $\mathrm{Xu}^{\prime}$ \\ Jiansong Ji $\mathbb{D}^{2}$ \\ Yongzhong Du'
}

'Institute of Pharmaceutics, College of Pharmaceutics Sciences, Zhejiang University, Hangzhou, 3 I0058, People's Republic of China; ${ }^{2}$ Department of Radiology, Lishui Hospital of Zhejiang University, Lishui, 323000, People's

Republic of China
Correspondence: Jiansong Ji

Department of Radiology, Lishui Hospital of Zhejiang University, Lishui, 323000,

People's Republic of China

Tel +86-578-22850II

Email Ischrjjs@I63.com

Yongzhong Du

Institute of Pharmaceutics, College of Pharmaceutics Sciences, Zhejiang

University, 866 Yu-Hang-Tang Road,

Hangzhou, 310058 , People's Republic of China

Tel +86-57I-8898I65I

Email duyongzhong@zju.edu.cn

\begin{abstract}
Benefiting from the rapid development of nanotechnology, photodynamic therapy (PDT) is arising as a novel non-invasive clinical treatment for specific cancers, which exerts direct efficacy in destroying primary tumors by generating excessive cytotoxic reactive oxygen species (ROS). Notably, PDT-induced cell death is related to T cell-mediated antitumor immune responses through induction of immunogenic cell death (ICD). However, ICD elicited via PDT is not strong enough and is limited by immunosuppressive tumor microenvironment (ITM). Therefore, it is necessary to improve PDT efficacy through enhancing ICD with the combination of synergistic tumor therapies. Herein, the recent progress of nanomaterials-based PDT combined with chemotherapy, photothermal therapy, radiotherapy, and immunotherapy, employing ICD-boosted treatments is reviewed. An outlook about the future application in clinics of nanomaterials-based PDT strategies is also mentioned.
\end{abstract}

Keywords: photodynamic therapy, immunogenic cell death, antitumor immunity, immunosuppressive tumor environment, nanomedicines

\section{Introduction}

Cancer remains becoming the top dominating reason for mortality in recent years with all the efforts in finding new treatment strategies and developing basic researches. For decades, the search for an effective cure for cancer is becoming relentless and urgent. Currently, surgery, radiotherapy, and chemotherapy are the most effective methods in the clinical treatment of cancers. ${ }^{1}$ Surgery is suitable for a few cancer patients in the early stage. Cancer patients, who cannot have surgery, always accept systemic radiotherapy and chemotherapy. However, radiotherapy efficacy is limited by the dose of the radiation, accompanying serious side effects. Chemotherapy is widely used for cancer patients. After having an initial response to chemotherapy, these patients usually have drug resistance, resulting in insignificant efficacy, increased mortality, and toxic effects.

Several novel proposed therapies that own their unique traits for treating cancers have been partly reported or adopted in preclinical researches such as immunotherapy, gene therapy, magnetic hyperthermia therapy, and phototherapy. Among all the novel therapies, photodynamic therapy (PDT) has absorbed much researches' attention, for it can directly kill cells without invasive injury. Based on a photosensitizer (PS) which can be activated by light at a relevant wavelength and causes singlet oxygen generation and irreversible cell death, PDT has been 
applied as a targeted therapy for cancers clinically for more than 40 years. ${ }^{2}$ PDT produces the singlet oxygen is effective in oxidizing biological substances such as nucleic acids, proteins, lipids in cells, and then damages the physiological structures of cells, disrupts cell metabolism and leads to cell apoptosis and necrosis. ${ }^{3}$ However, there are still many challenges for broad application of conventional PDT in a clinic, such as most of the PS lacks the tumor-targeting ability, ${ }^{4}$ limited light penetration towards tumor tissues, ${ }^{5}$ hypoxia tumor microenvironment, ${ }^{6}$ limited PS accumulation ${ }^{7}$ and T cell infiltration in tumors. ${ }^{8,9}$ Benefiting from the development of nanotechnology, advanced nanomaterials enable PDT to overcome these difficulties step by step and expand the range of application, achieving a satisfactory antitumor treatment effect.

Notably, the process of PDT inducing cell apoptosis and necrosis can effectively trigger immunogenic cell death (ICD), which is an important phenomenon that results in the improving immunogenicity of the tumor microenvironment (TME). Finally, ICD elicited by PDT can trigger systemic antitumor immunity via $\mathrm{T}$ cell responses. Fong et al succeeded to eliminate about $70 \%$ of tumors by PDT treatment with a durable antitumor immunity for more than 1 year in a CT26 murine model. ${ }^{10}$ Nevertheless, recent researches further point out that PDT-caused antigen-specific antitumor immunity is insufficient to eradicate tumor residues and inhibit metastasis. ${ }^{11}$ This phenomenon attributes to the ICD elicited by PDT is not strong enough and ICD is always limited by the immunosuppressive tumor environment (ITM). Thus, researchers focus on the development of nanomaterials-based PDT with boosted ICD efficacy in recent years. This review introduces the recent advances of nanomaterials-based PDT and its mechanism of triggering ICD in detail. Then the main approaches of amplifying PDT efficacy in the induction of ICD are discussed. Finally, we reviewed the rational methods to improve the induction of ICD in PDT-based anticancer therapies, such as the combination of chemotherapy, photothermal therapy, radiotherapy, and immunotherapy.

\section{Nanomaterials-Based Photodynamic Therapy}

More than 100 years ago, the researcher discovered that a combined approach of certain chemicals and light could lead to cell death. With steady development and continuous research, this technique is widely used in clinical treatment, which is called PDT. ${ }^{12}$ PDT, as an invasive treatment, exerts its effects by applying PS to generate excessive reactive oxygen species (ROS) under suitable light irradiation.

In simple terms, the PS molecule is in a singlet ground state $\left(\mathrm{S}_{0}\right)$ normally. As shown in Figure 1, with the irradiation of proper light, the PS is activated to reach an excited singlet state $\left(\mathrm{S}_{1}\right)$. The $\mathrm{S}_{1}$ state is stabilized either by emitting fluorescence or by a non-radiative process via intersystem crossing from a singlet to a triplet state $\left(\mathrm{T}_{1}\right)$. The PS in the $\mathrm{T}_{1}$ state

\section{Excited singlet state}

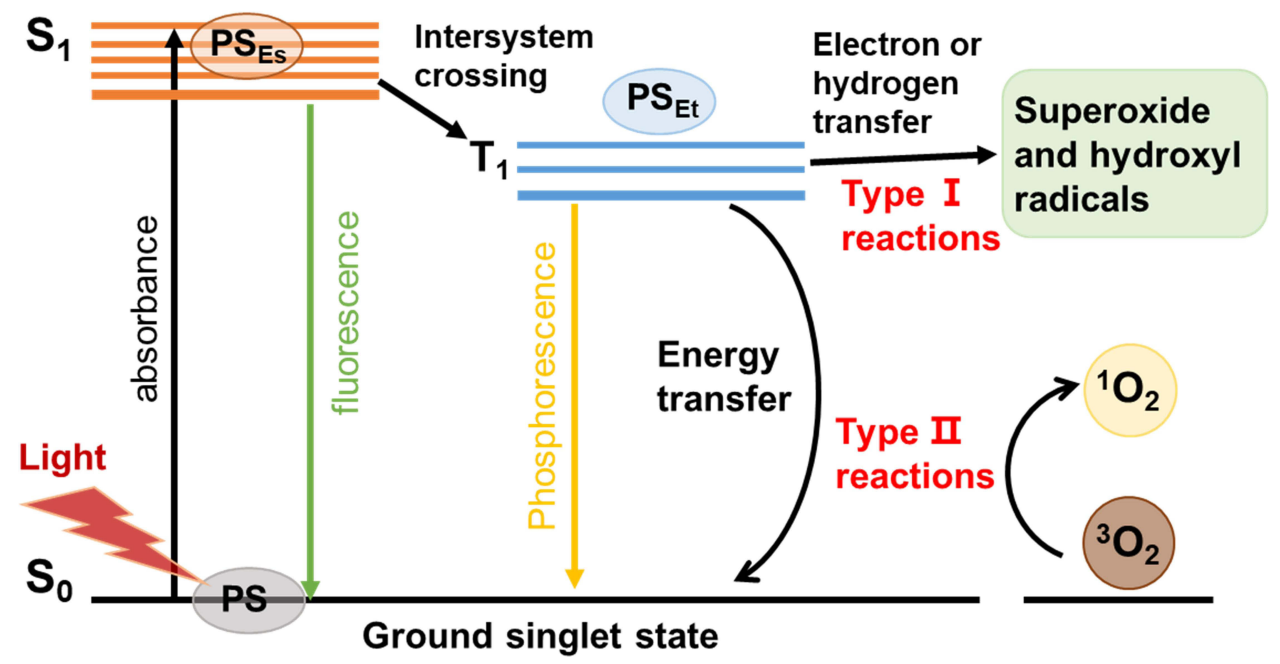

Figure I Molecular mechanism of PDT. With the light irradiation, PS is activated from the ground singlet state $\mathrm{S}_{0}$ to the exited singlet state $\mathrm{S}_{1}$. By intersystem crossing, they are converted to the excited triplet state $T_{1}$. Afterwards, electron transfer from $T_{1}$ to biological substrates (type I reaction), or energy transfer to molecular oxygen (type II reaction) that leads to a burst of ROS production. 
can produce cytotoxic ROS through Type I and Type II reactions. The Type I reaction involves the transfer of either a hydrogen atom or an electron between the excited PS and the substrates, leading to the generation of free radicals. These radicals then react with oxygen, resulting in the production of ROS such as superoxide $\left(\mathrm{O}_{2} \bullet\right)$, hydroxyl radicals $(\mathrm{HO} \bullet)$ and hydrogen peroxide $\left(\mathrm{H}_{2} \mathrm{O}_{2}\right)$. The Type II reaction involves the energy transfer between the excited PS and the molecular oxygen in the ground state $\left({ }^{3} \mathrm{O}_{2}\right)$, resulting in the formation of a highly reactive state of oxygen known as singlet oxygen $\left({ }^{1} \mathrm{O}_{2}\right) .{ }^{13,14}$

The excessive ROS generated by PS is efficient in killing cancer cells, which could directly destroy cellular organelles (eg mitochondria, cell nucleus, lipids). When treating proper laser irradiation, PDT has been applied in various cancers, such as skin, prostate bladder, head, neck, and lung cancers. In a clinic, when patients receive PDT treatment, they would be injected with PS firstly, and then the treatment sites are irradiated with specific light, resulting in the consumption of oxygen and generation of cytotoxic ROS, which leads to tissue destruction and cell death. $^{15}$ Three vital elements: PS, light, oxygen offer equal efficacy to the PDT, while every single element has no effects. Compared to traditional tumor therapies including surgery, radiotherapy, chemotherapy, PDT owns its unique characteristics, such as lower toxicity of normal tissues, sufficient efficacy, and accurate targeting treatment. $^{16}$ Therefore, PDT is emerging as a prospective method for intractable cancer treatment in the clinic.

However, PDT has not yet become a first-line intervention method of clinical cancer treatment, and this is mainly caused by several reasons: (1) the commonly used PS is hydrophobic molecules, which lack stability and selectivity for tumor cells in the biomedical system. ${ }^{17}$ (2) The treatment tissue depth of PDT clinically is relatively low because the relevant light PS absorbed exists as short wavelength, which fails to penetrate the deep tissues. ${ }^{5}$ (3) In the process of photodynamic reaction, the PS needs to transfer energy to oxygen to generate highly efficient ROS. And excessive ROS makes PDT have serious oxygen dependence. Tumor hypoxia is confirmed as a unique trait of most solid tumors, largely limiting the efficacy of PDT. $^{18}$

To overcome the above shortcomings, improve and broaden the clinical application of PDT, more and more tumor-targeting drug delivery system has been widely developed. Benefiting from nanotechnology, recent reported nanomaterials-based PDT overcomes above shortages and the application of PDT is expanded. Qian et al reported tumor-targeting theranostics nanoparticles loaded with PS (Ce6), called HSA-Ce6@HSA-RGD for image-guided PDT. ${ }^{19}$ The self-assembled RGD modified nanoparticles (NPs) could effectively deliver Ce6 to tumor sites, which overcame the tumor-targeting limitation of PS. After laser irradiation, the treated mice's overall survival lengthened to 40 days without any mice death. To solve PDT's limited penetrating ability, researchers applied lanthanide-doped upconversion nanoparticles (UCNPs) to form UCNPs-based PDT, which is triggered by nearinfrared light (NIR). Benefiting from the long wavelength, NIR light can penetrate deep tissues $(>3 \mathrm{~cm})$ with lower light scattering and absorption of human tissues. Besides, NIR light can be absorbed by UCNPs to elicit visible or UV light. A UCNP-based micelle, which can transfer NIR light to $540,650 \mathrm{~nm}$ luminescence, was created for tumor imaging and PDT by Gong et al. ${ }^{20}$ Rose Bengal (RB) molecules as PSs could absorb $540 \mathrm{~nm}$ luminescence and generate ROS for effective PDT. In addition, $650 \mathrm{~nm}$ luminescence was captured for specific tumor imaging. Furthermore, the design of oxygen-carrying nanocarriers could safely and efficiently transport oxygen to the tumor site, improve the hypoxia state of the tumor sites, and solve the serious problem of oxygen dependence in PDT. Besides, Guoliang et al reported an oxygen self-sufficient NIRresponsive nanosystem for enhanced PDT against hypoxic tumors, which had high oxygen capacity and showed remarkable efficacy of PDT. ${ }^{21}$ In general, the mainstream applied nanomaterials to deliver PS for better PDT efficacy in vivo, mainly including liposomes, synthetic micelles, polymer nanoparticles and inorganic nanostructures. Organic nanomaterials including liposomes, micelles and polymers can be modified with tumor-targeting molecules, which not only improve the stability and biocompatibility of PS but also deliver PS to the target cells to improve the curative effect and reduce side effects. However, the limited tissue penetrating ability of PDT remains unsolved. And the recent reported functional nanomaterials, especially the inorganic materials, such as UCNPs, quantum dots, carbon nanomaterials and gold nanomaterials, which can be used as NIR light acceptors to activate PS and result in tumor cell damage in deep tumor treatment directly. ${ }^{22}$ Although the inorganic nanoparticles face the challenge of biocompatibility in vivo, their unique multifunctional ability enables them to be promising candidates in the field of deep tumor PDT. Therefore, taking full advantage of nanotechnology, 
nanomaterials-based PDT exhibits specific tumor targeting and accumulating ability, that provides deeper treatment depth with reduction of side effects, and achieves much ideal efficacy in clinical therapeutic applications than conventional PDT.

\section{PDT Induces Immunogenic Cell Death}

ICD is an umbrella term covering several cell death modalities, such as apoptosis, necroptosis and ferroptosis. Although these cell death modalities differ from each other morphologically and biochemically, they are all characterized by the emission of immune-stimulatory molecules. ${ }^{23}$ Immunogenic apoptosis is characterized by ROS-based endoplasmic reticulum (ER) stress and induction of autophagy, and its immunogenicity is caspase-dependent. Notably, PDT induces a specific form of immunogenic apoptosis. ${ }^{24}$ Necroptosis is the cell death mode that can be blocked by inhibitors of RIPK1, RIPK3 and MLKL, which is usually immunogenic. ${ }^{25}$ Ferroptosis is an iron- and ROS-dependent form of regulated cell death. ${ }^{26}$ Recently, ferroptosis was reported to induce an anti-tumor immune response for tumor therapy. ${ }^{27}$

Recent researches demonstrated that PDT can dramatically activate adaptive and innate antitumor immunity. In the direct pathway, PDT gives rise to acute inflammatory responses by oxidative stress and local damages, which leads to activation of innate immunity. In the indirect pathway, tumor cells destroyed by the PDT procedure would elicit the adaptive immune responses through triggering damage-associated molecular patterns (DAMPs) and the release of tumor cell debris during the ICD process. ${ }^{28}$ The activation of DAMPs is essential to ICD, including the expression of calreticulin (CRT) on the cell membrane surface, and extracellular secretion of high-mobility group box 1 (HMGB1) and adenosine triphosphate (ATP). ${ }^{29}$ In detail, CRT is known as an "eat me" signal accompanying HMGB1 and ATP secretion, which can activate antigen presentation cells (APCs) such as dendritic cells (DCs) to exert antitumor immunity. ${ }^{30}$ Besides, exogenous HMGB1 can interact with Toll-like receptors on DCs, which promotes proinflammatory factor generation, thereby inducing the maturation of DCs. Mature DCs voluntarily migrate to lymph nodes and present specific antigens to primary T cells. ${ }^{29,31}$ And then, activated cytotoxic $\mathrm{T}$ lymphocytes (CTLs) efficiently infiltrate into tumor sites, leading to eliminate the primary tumor and track metastatic tumor sites. $^{32}$ The illustration of PDT induces $\mathrm{T}$ cell-mediated antitumor immunity via ICD was shown in Figure 2 . It is worth noting that DAMPs are the main characteristics of cancer cells undergoing ICD, which are associated with induction of an anti-tumor immune response. ${ }^{33}$ However, ex vivo measurements of ICD parameters are potentially artifact-prone. Thus, the "gold standard" protocol for the in vivo assessment of ICD in mice should be applied, which includes the experiment of tumor prophylactic vaccination, followed by rechallenge with living entities of the same type, in syngeneic immunocompetent animals. ${ }^{34}$ The initial research was reported in 1994, Canti and his colleagues compared the efficacy of normal mice and

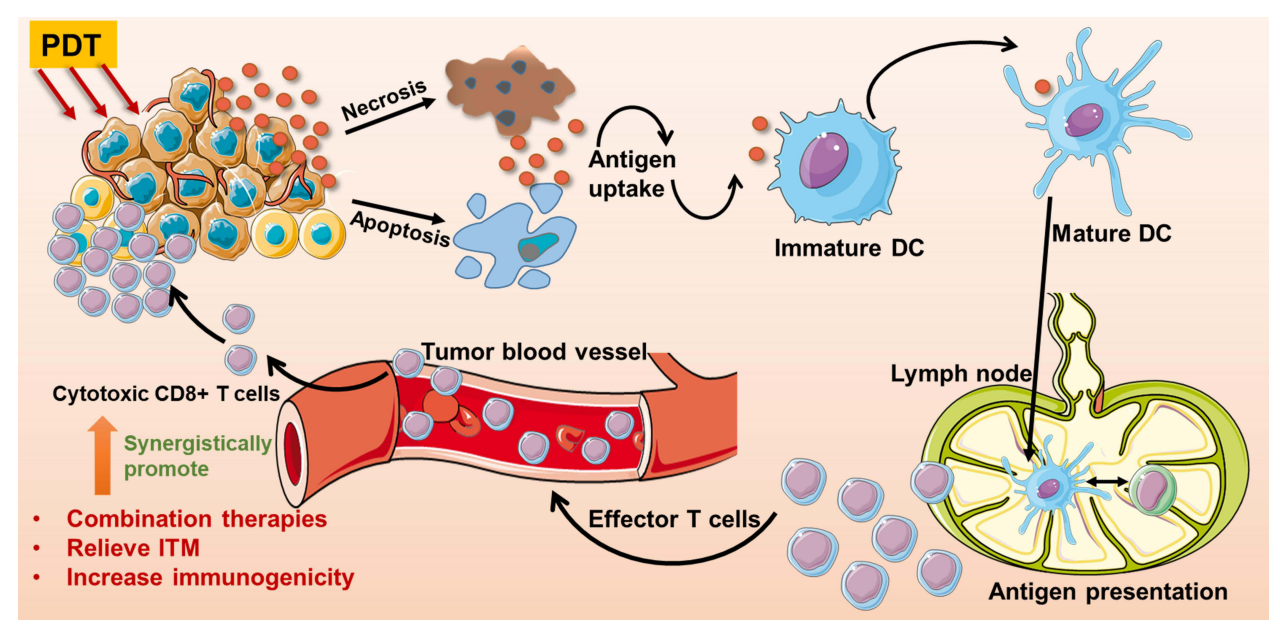

Figure 2 The process of PDT-triggered antitumor T cell responses via immunogenic cell death and the rational strategies to improve antitumor immune responses. PDT killed cells can release cytokines, which stimulate DCs' maturation. Mature DCs spontaneously migrate to lymph nodes and present antigens to T cells. After antigenpresenting, effector T cells efficiently migrate to tumor sites and kill cells. The rational strategies of promoting PDT-triggered antitumor immunity include combined PDT with ICD-boosted therapies, decrease immunoregulatory suppression with ITM relief, and increase tumor immunogenicity. 
immunodeficiency mice bearing MS-2 fibrosarcoma treated with long-term PDT. The results demonstrated that the survival rate of all mice was significantly improved, and mice with normal immunity had no tumor recurrence, while tumor recurrence was observed in immunodeficiency mice. $^{35}$ Therefore, through ICD-mediated immune responses, PDT offers a spontaneous antitumor immunity with local release of DAMPs and mature DCs, finally leading to activation of cytotoxic $\mathrm{T}$ cells for tumor metastasis and proliferation.

However, recent studies have pointed out that even if PDT causes antigen-specific $\mathrm{T}$ cell immune responses, these antitumor immune responses are not strong enough to eradicate tumor residues. $T$ cell responses induced by ICD are widely limited in vivo. On one hand, ICD initiated by PDT is relatively weak and short-lived, which cannot support systematic $\mathrm{T}$ cell responses. On the other hand, an autologous immune response is limited by immunosuppressive tumor microenvironment (ITM), which is the reason why most conventional PDT did not exhibit expected long-lived antitumor efficacy. ${ }^{36}$ Thus, to improve the antitumor efficacy of nanomaterials-based PDT, it is vital to offer proper approaches to boost ICD and rationally reverse ITM. To overcome the limitation of ICD induced by PDT, there are 3 rational strategies shown in Figure 2: (1) combination of PDT with other ICD-based therapies, including radiotherapy, chemotherapy, which increase the release of ICD inducers, and amplify ICD efficacy; (2) decrease immunoregulatory suppression with ITM reversion, such as applying immune checkpoint inhibitors to combine with PDT; (3) enhance the immunogenicity of tumor cells, leading to the maturation of DCs, such as utilizing immunoadjuvants during the PDT treatment. Herein, recent representative nanomaterials-based researches which improve PDT efficacy via the combination of ICD-amplified therapies with boosted antitumor immunity have been reviewed in detail.

\section{Combination of PDT with ICD-Based Therapy}

Recently, several potential therapies such as phototherapy, chemotherapy, and radiotherapy, are effective in eliciting ICD. The combination of PDT with these ICD-based cancer therapies can synergistically induce DCs maturation and elicit cytotoxic T cells, which has a great potential for treating metastatic cancers without effective treatments. ${ }^{37}$
Advanced nanotechnology enables scientists to create a tumor-targeting drug delivery system for safe, effective, and compatible combined therapy.

\section{Combination of PDT with Chemotherapy}

Chemotherapy is the first-line treatment in clinical cancer therapy with wide application. Recently, it has been proved that some chemotherapeutic agents including oxaliplatin (OXA), ${ }^{38}$ doxorubicin (DOX), ${ }^{33}$ and mitoxantrone, had antitumor immunity through provoking ICD in the TME. Nanomaterials-based drug delivery system, which co-delivers PS and chemotherapeutic drugs, can realize synergistic efficacy of PDT and chemotherapy, with increased tumor accumulation and precise release at tumor sites. On one hand, combining PDT with chemotherapy can overcome the multidrug resistance (MDR) produced during tumor treatment and reduce the therapeutic dose of chemotherapeutic drugs. ${ }^{39}$ On the other hand, PDT combined with chemotherapy increases the release of DAMPs, enhances ICD efficacy, and thereafter triggers CTLs infiltration in tumor sites for tumor regression.

He and colleagues successfully synthesized core-shell nanoparticles called NCP, which co-delivered PS pyrolipid and OXA for combination therapy of PDT and chemotherapy. Pyrolipid-triggered PDT with OXA releasing induced immune responses, resulting in the inhibition of distant tumors in bilateral syngeneic mouse tumor models of CT26 and MC38. The results of in vitro experiments demonstrated NCP@pyrolipid with light irradiation could induce ICD with secretion levels of CRT, IFN- $\gamma$, IL-6, TNF- $\alpha$ significantly increased. ${ }^{40} \mathrm{Ni}$ et al reported the application of a mental-organic framework (MOF) nanoparticle-loaded $\mathrm{Cu}$-porphyrin with antitumor efficacy of PDT and hormone-induced chemotherapy. The nanoplatform was specifically designed for hormone dysfunction tumor subtypes. The results demonstrated that $\mathrm{Cu}$-TBP with light had a significantly stronger ICD compared to other treatments for synergy of hormone-induced chemotherapy and PDT process. ${ }^{41}$ A melanoma mouse model of single B16F10tumor bearing $\mathrm{C} 57 \mathrm{BL} / 6$ mice was used to assess the in vivo treatment efficacy, which showed the tumor growth inhibition indice was about $96.6 \%$ of the $\mathrm{Cu}$ TBP plus light irradiation.

DOX is emerging as a practical agent for ICD induction. $^{42}$ A high-density lipoprotein-mimicking nanodiscs loaded with DOX was applied with immunotherapy for preclinical researches. Combined chemoimmunotherapy 
with nanodiscs plus anti-programmed death 1 therapy resulted in 80 to $88 \%$ of survivors in both MC38 and CT26 colon murine models. ${ }^{43}$ The combination of DOX and PDT not only provokes cytotoxic ROS but also reverses ITM via inducing ICD and modulating immune cytokines, which significantly improve chemo-photodynamic therapy efficacy. ${ }^{44}$ Jin et al designed a multifunctional UCNP coloaded with PS RB and DOX, for chemo-photodynamic therapy upon NIR irradiation. In vitro and in vivo research confirmed DOX and ROS released by PDT both induce ICD with overexpressed CRT and enhanced secretion of ATP, HMGB1. Results also indicated that chemo-PDT treatment was effective in inhibiting tumor metastasis in lungs with an increased proportion of $\mathrm{CD}^{+}, \mathrm{CD}^{+} \mathrm{T}$ lymphocytes in a $4 \mathrm{~T} 1$ murine model. ${ }^{45}$

\section{Combination of PDT with Photothermal}

\section{Therapy}

Photothermal therapy (PTT) is a kind of hyperthermia cure for tumors via the usage of chemical agents that can absorb light and elicit hyperthermia efficacy. ${ }^{11}$ During PTT treatments, the local high temperature in tumor sites causes large amounts of cell death and induces different biological reactions, especially the release of DAMPs. PTT results in the overexpression or enhanced secretion of heat shock proteins (HSPs), HMGB1, and ATP. ${ }^{46,47}$ However, hyperthermia has obvious damage in surrounding normal tissues, resulting in the inactivation of immune effective proteins, thereby causing suppression of host antitumor immunity. ${ }^{48}$ PTT and PDT are known to be the two main noninvasive medical techniques in treating various diseases including tumors. The combination of PTT and PDT not only triggered the photothermally enhanced PDT efficiency, but also induced a synergistic effect - the photothermal effects can accelerate intratumoral blood flow, thus leading to more oxygen transported into the tumor to amplify the PDT efficacy. ${ }^{49,50}$ In addition, PTT and PDT all belong to phototherapy, and they all exhibit a $\mathrm{T}$ cell helper 1 (Th1)-based immune response. Thus, the combination of PDT and PTT triggered by a single light source is widely investigated. As shown in Figure 3, chemical molecules absorbed relevant wavelength light to exert antitumor effects, via biochemical reaction of PTT, PDT, and photoimmunotherapy (PIT). Checkpoint inhibitors applied in phototherapy are supposed to block immune checkpoint receptors for enhancing cytotoxic efficacy of antigen-specific T lymphocytes. ${ }^{51}$

For instance, Wang et al reported a UCNP nanoplatform constructed by self-assembled indocyanine green (ICG) and DSPE-PEG, which loaded PS. ICG significantly

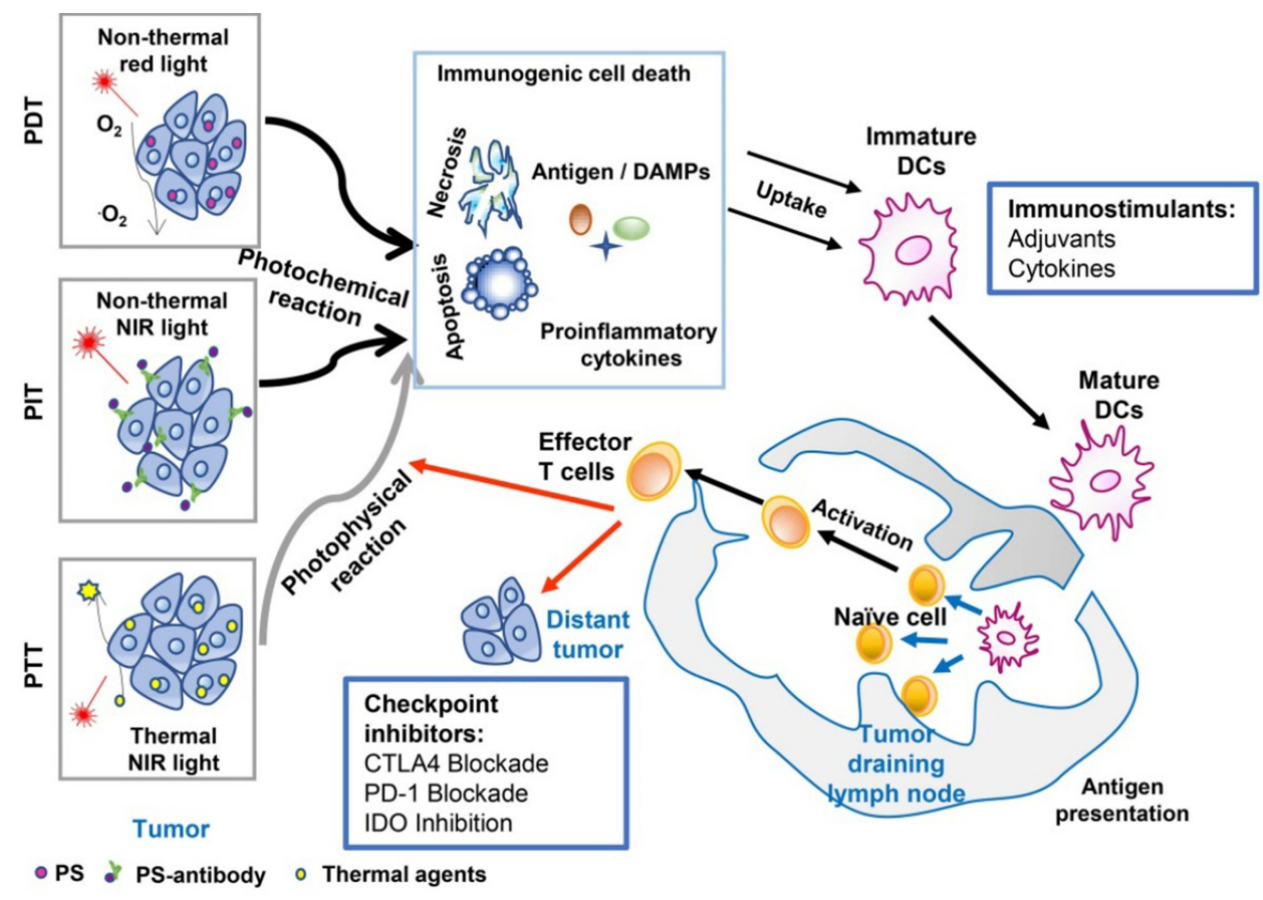

Figure 3 An overview of cancer treatment using the combination of phototherapy and Schematic depiction of the combined therapy of immunotherapy and phototherapy. Note: Reproduced from Wang M, Rao J, Wang M, et al. Cancer photo-immunotherapy: from bench to bedside. Theranostics. 202I;II(5):22I8-223I. doi:I0.7I50/ thno.53056. ${ }^{51}$ 
enhanced PDT efficiency and synchronously enabled selective PTT upon NIR irradiation. The results demonstrated that UCNPs co-loaded PS, ICG phototherapy strategy exhibited an enduring immune memory response in an orthotopic breast cancer model of $4 \mathrm{~T} 1$ in BALB/c mice, which protected tumor-bearing mice from tumor relapse. ${ }^{52}$ Accompanied with a checkpoint inhibitor, there were approximately $84 \%$ of mice survived over 2 months, and over $34 \%$ of mice developed antitumor immunity. These findings imply that PDT combined PTT with a checkpoint inhibitor could effectively elicit systemic T cell activation.

Recent researches demonstrated ROS evoked ICD via triggering ER stress. As ER stress is important in activating ICD, researchers built an ER-targeting drug delivery system for improving PDT efficacy. ${ }^{53}$ The gold nano-spheres consisted of ER-targeting peptides and ICG (FAL-ICG-HAuNS) was designed by Li et al The nanoparticles can generate an ERtargeting ability in realizing PDT and PTT. ${ }^{54}$ Meanwhile, they applied hemoglobin $(\mathrm{Hb})$ liposomes to deliver oxygen in vivo, which were efficient in reversing tumor hypoxia and evoking PDT efficacy. The experiments taken in vivo depleted activated $\mathrm{T}$ lymphocytes via injecting anti-CD4 and CD8 antibodies in CT-26 and B16 tumor models, which was supposed to figure out the dominant role in PDT/PTT-mediated immunotherapy. The results proved that ICD-mediated cytotoxic $\mathrm{T}$ cell responses owned a dominant role in PDT/PTT-triggered antitumor immunity. Taken together, ER-targeting PDT combined with PTT exhibited an outstanding efficacy of combating primary and distant tumors accompanied with a strong longterm antitumor immunity.

\section{Combination of PDT with Radiotherapy}

Benefiting from the deep tissue penetration, X-rays have been broadly applied in clinics for cancer imaging and curing. Besides, radiotherapy (RT) is existing as a powerful widespread cancer treatment with over $70 \%$ of cancer patients' adoption for more than 100 years. ${ }^{55}$ The mechanism of RT inducing cell death is believed to be the DNA damage of X-rays, which can also evoke ICD in situ and stimulate T cellmediated anticancer effects. ${ }^{56}$ However, RT is still facing the challenges created by its severe adverse effects on normal organs and radio-resistance of tumor cells. ${ }^{57}$ Relevant studies have shown that PDT combined with RT can increase the sensitivity of tumors to radiation and improve the effectiveness of treatment. ${ }^{58}$ At the same time, PDT can also shorten the exposure time or reduce the radiation dose of RT. In clinical research, it was confirmed that PDT combined RT for patients with advanced tumors could significantly improve the quality of life, alleviate the symptoms, relieve pain and prolong survival. ${ }^{59}$

Recently, the scintillator is widely investigated, which is a kind of material that can transfer X-rays to UV-visible luminescence for X-ray-induced PDT after loading various PS, for a combination of PDT with RT. Many types of researches have focused on PS-loaded nanoplatforms to transfer X-rays into proper luminescence, achieving combined antitumor effects of PDT and RT with a lower dosage of X-ray irradiation. $^{60,61}$ For example, Sun and colleagues created a PSloaded heterogenous Au nanoplatform named AIE-Au, which enabled a lower dosage of X-ray-induced PDT with fewer adverse effects. $^{62}$ The outcomes in research confirmed that AIE-Au could not only produce excessive ROS with a dramatic decrease of X-ray dosage but exert effective RT efficacy in U87MG tumor-bearing mice in vivo. Besides, Doix et al used cancer cells killed by PDT to stimulate DCs and then assessed the combined antitumor efficacy of stimulated DCs and RT in vivo. ${ }^{63}$ The researchers applied PS OR141 as an efficient ICD inducer with a relatively low dosage to achieve maximal antitumor immune responses. Matured DC activated by PDT, was first injected in tumor-bearing mice for a period, and then those mice received RT treatment, resulting in a significant delay of tumor proliferation. The results illustrated that it was crucial for combining T-cell mediated immune responses with RT treatments. Further, these researches explored RT combined PDT with an immune checkpoint inhibitor for mesothelioma cancer treatment. ${ }^{64}$ The in vivo results showed an increased proportion of $\mathrm{CD}^{+}$and $\mathrm{CD}^{+} \mathrm{T}$ cell infiltrating in tumor sites and the significant enhancement of cytokine release such as IFN- $\gamma$ and TNF- $\alpha$.

\section{Combination of PDT with Other Therapies}

In recent years, more and more new therapies were synergistically applied with PDT for better systemic antitumor efficacy. Adoptive cell therapy (ACT) is a kind of immunotherapy in which $\mathrm{T}$ cells are genetically modified to express a chimeric antigen receptor (CAR) or $\mathrm{T}$ cell receptor (TCR). ${ }^{65}$ During the ACT treatment process, large numbers of tumor-specific $\mathrm{T}$ cells are obtained from patients and expanded in vitro and infused back to patients. To some extent, ACT strengthens or alters the intrinsic immune capacity and exploits its efficiency in the treatment of cancer disease. However, ACT has had limited success in the treatment of solid tumors due to the protective microenvironment. ${ }^{66}$ In preclinical researches, many researchers have explored the combination strategies to overcome the limited efficacy of ACT. The combination 
of CAR-T and PTT has been applied and showed superior CAR-T accumulation and tumor-control efficacy in solid tumor models. ${ }^{67}$ However, the research about CAR-T combined with PDT has not been reported so far. Besides, Mladen Korbelik reported the application of a human natural killer (NK) cell line which was genetically altered to produce interleukin-2 (IL-2) as the adjuvant after PDT treatment. The modified NK cells were used for adoptive immunotherapy in combination with PDT and the results demonstrated their effectiveness in improving the outcome of therapy of both subcutaneous xenografted human tumors in immunodeficient mice and mouse tumors growing in syngeneic immunocompetent mice. ${ }^{68}$ Recently, Fang et al designed PDT-motivated autologous tumor cell-based vaccines (P-ATVs), which could be easily produced within a few days and efficiently boost neoepitope-specific $\mathrm{CD} 8^{+} \mathrm{T}$ cells to activate adoptive immunotherapy, unlike ACT or neoantigen-based vaccine that takes several months to prepare. ${ }^{69} \mathrm{P}$-ATV was engineered by coating oxidized autologous tumor cells with polyethylenimine-conjugated chlorin e6 (PEI-Ce6) in hydrogels. The results demonstrated that modified tumor cells coupled with PDT could effectively mature DCs, suppress regulatory $\mathrm{T}$ cells, boost neoepitopespecific $\mathrm{CD}^{+} \mathrm{T}$ cells, and inhibit tumor relapse in melanoma and colorectal mouse models without obvious side effects.

Sonodynamic therapy (SDT) as a new non-invasive approach developed from PDT, it can kill tumor cells selectively and specifically. Similar to PDT, the scientific basis of SDT-induced toxicity involves the generation of ROS by the combination of low-intensity ultrasound, $\mathrm{O}_{2}$, and sonosensitizers. ${ }^{70}$ Both ultrasound and sonosensitizers are nontoxic. Moreover, deep tissue penetration of ultrasound waves allows the destruction of deep-seated or large tumors. ${ }^{71}$ Preclinical animal models have confirmed that the dying tumor cell fragments induced by the SDT procedure can be used as the source of tumor antigens to trigger the host antitumor immune efficacy. ${ }^{72}$ Therefore, SDT combined with PDT can not only overcome the poor tissue penetration of PDT, but can also induce systemic antitumor immunity. Zheng et al synthesized a UCNP-based platform co-loaded with protoporphyrin (PpIX) and DOX for achieving a good tumor therapeutic efficacy. The PS as well as sonosensitizer PpIX exhibits a response to both ultrasound and NIR stimulation. B16-F0 tumor model was established for in vivo SDT and PDT studies. ${ }^{73}$ The most severe tumor cell damage was presented in the synergistic treatment group, while cancer cells in other groups were not notably affected or only showed partial damage and apoptosis. Similarly, Liu et al developed a selfassembled RB-loaded peptido-nanomicelles (RBNs), and they were used as both engineered molecular probes and a novel nano-formulation for synergy-enhanced tumor treatment of SDT, PDT and chemotherapy. ${ }^{74}$

\section{Strategies of Relieving Immunoregulatory Suppression}

Although PDT triggers antitumor immunity via the ICD process, its efficacy is not strong enough to eradicate tumor residuals under an ITM. The unique TME is reported as one of the most important parts of tumor therapies, which is tightly related to tumor metastasis and recurrence. Thus, ITM efficiently limits PDT-induced ICD efficacy. Besides, PDT triggered ROS prompts oxidative reaction of danger signals, which induces immune suppression or tolerance with immunosuppressive cytokine release. ${ }^{11}$ Therefore, to counteract the immunosuppressive effects of PDT and decrease immunoregulatory suppression with ITM reversion, more and more researchers focused on PDT combined with immune checkpoint blockade (ICB). ICB directly blocks immunosuppressive receptors and breaks tumor immune suppression, leading to the strengthened cytotoxic $\mathrm{T}$ cells and unleashes the therapeutic potential of endogenous antitumor immune responses. ${ }^{75}$ Thus, when ICB is combined with PDT, it can inhibit the immune checkpoint activity in the TME, promoting PDTinduced antitumor immune responses. Meanwhile, PDT can lead to the release of DAMPs and tumor antigens that are essential to triggering an innate immune response, thereby serving as a good adjuvant to immunotherapy. These factors could serve to promote synergy between PDT and immune checkpoint blockade. ${ }^{76}$

Currently, the main ICB therapy using indoleamine 2,3-dioxygenase (IDO), anti-programmed death-ligand 1 $(\alpha \mathrm{PD}-\mathrm{L} 1)$, anti-programmed death 1 ( $\alpha \mathrm{PD}-1)$, and anticytotoxic T lymphocyte-associated antigen 4 ( $\alpha$ CTLA- 4 ) antibodies have exhibited great capability in clinics. Treated antibodies can block IDO, ${ }^{77}$ CTLA- $4,{ }^{78}$ or PD$1,{ }^{79}$ and dramatically lengthen the overall survival of various cancer patients. Thus, the strategy of combined ICB therapy with nanomaterials-based PDT is a promising approach and attracts lots of researchers' attention in recent years. The recent researches of nanomaterialsbased PDT combined with ICB were tabulated in Table 1. 
Table I Preclinical Studies of Nanomaterials-Based PDT Combined with ICB

\begin{tabular}{|c|c|c|c|c|c|c|c|}
\hline PS & $\begin{array}{c}\text { Mediated } \\
\text { Nanomaterials }\end{array}$ & $\begin{array}{c}\text { ICB } \\
\text { Receptor }\end{array}$ & $\begin{array}{l}\text { Combined } \\
\text { ICB Drug }\end{array}$ & $\begin{array}{l}\text { Light } \\
\text { Source }\end{array}$ & $\begin{array}{l}\text { Cancer } \\
\text { Model }\end{array}$ & Effectiveness & Ref \\
\hline H4TBC & MOF & IDO & INCB24360 & $650 \mathrm{~nm}$ & $\begin{array}{l}\text { CT26 and } \\
\text { MC38 } \\
\text { tumor } \\
\text { models }\end{array}$ & $\begin{array}{l}\text { This treatment promoted an efficient } \\
\text { inhibition in local and abscopal tumors in } \\
\text { a bilateral tumor murine model. }\end{array}$ & [84] \\
\hline Porphyrin & $\begin{array}{c}\text { Redox-activatable } \\
\text { liposome }\end{array}$ & IDO & NLG-8I89 & $660 \mathrm{~nm}$ & $\begin{array}{l}4 \mathrm{TI} \\
\text { tumor } \\
\text { murine } \\
\text { model }\end{array}$ & $\begin{array}{l}\text { The percentage of } \mathrm{CD}^{+} \mathrm{T} \text { cells in the } \\
\text { treated tumors significantly enhanced to } \\
\text { approximately } 10.51 \% \text {, compared to that } \\
\text { in PBS group was about } 5.11 \% \text {. }\end{array}$ & [85] \\
\hline Ce6 & $\begin{array}{l}\text { iPSs (self-assembly of } \\
\text { Ce6 and NLG919) }\end{array}$ & IDO & NLG919 & $660 \mathrm{~nm}$ & $\begin{array}{l}\text { CT26 } \\
\text { tumor } \\
\text { murine } \\
\text { model }\end{array}$ & $\begin{array}{l}\text { The percentages of } \mathrm{CD} 4^{+} \text {and } \mathrm{CD} 8^{+} \\
\mathrm{T} \text { cells in PDT combined immunotherapy } \\
\text { achieved } 2.3,5.1 \text {-fold higher than that in } \\
\text { ICB treatment, which was } 1.4 \text {, I.8-fold } \\
\text { higher than the PDT treatment. }\end{array}$ & [86] \\
\hline Ce6 & UCNPs & CTLA-4 & $\begin{array}{l}\text { Anti-CTLA4 } \\
\text { antibody }\end{array}$ & $980 \mathrm{~nm}$ & $\begin{array}{l}\text { CT26 } \\
\text { tumor } \\
\text { murine } \\
\text { model }\end{array}$ & $\begin{array}{l}\text { PDT applied with } \alpha \text { CTLA- } 4 \text { provided an } \\
\text { outstanding effect in eradicating residual } \\
\text { tumors with enduring immune responses, } \\
\text { especially curing distant tumors without } \\
\text { direct light irradiation. }\end{array}$ & [88] \\
\hline $\mathrm{ZnPc}$ & UCNP@SiO2@AuNPs & CTLA-4 & $\begin{array}{l}\text { Anti-CTLA4 } \\
\text { antibody }\end{array}$ & $980 \mathrm{~nm}$ & $\begin{array}{l}4 \mathrm{TI} \\
\text { tumor } \\
\text { murine } \\
\text { model }\end{array}$ & $\begin{array}{l}\text { The treatment was effective in inhibiting } \\
\text { the subcutaneous xenograft tumors with } \\
\text { minor side effects. }\end{array}$ & [89] \\
\hline Ce6 & M-MONs & CTLA-4 & $\begin{array}{l}\text { Anti-CTLA4 } \\
\text { antibody }\end{array}$ & $660 \mathrm{~nm}$ & $\begin{array}{l}\text { MCF-7 } \\
\text { and } 4 \mathrm{TI} \\
\text { tumor } \\
\text { murine } \\
\text { model }\end{array}$ & $\begin{array}{l}\text { The treatment system-induced ICD } \\
\text { including DAMPs release, TNF- } \alpha \text {, IL- } 6 \text {, } \\
\text { and IFN- } \gamma \text { secretion, which promoted } \\
\text { DCs maturation, CTLs infiltration, and } \\
\text { reduced Tregs activation. }\end{array}$ & [90] \\
\hline Pyrolipid & Zn-pyrophosphate & PD-LI & $\begin{array}{l}\text { Anti-PD-LI } \\
\text { antibody }\end{array}$ & $670 \mathrm{~nm}$ & $\begin{array}{l}4 \mathrm{TI} \\
\text { tumor } \\
\text { murine } \\
\text { model }\end{array}$ & $\begin{array}{l}\text { The combined application of PDT and } \\
\alpha \mathrm{PD}-\mathrm{LI} \text { resulted in a } 92 \% \text { reduction in } \\
\text { tumor volumes compared to the PBS } \\
\text { group, indicating a satisfactory efficacy for } \\
\text { metastatic tumors. }\end{array}$ & [93] \\
\hline Ce6 & $\begin{array}{l}\text { Ce6/BMS-202 } \\
\text { nanoparticles }\end{array}$ & $\begin{array}{l}\text { PD-I/PD- } \\
\text { LI }\end{array}$ & BMS-202 & $660 \mathrm{~nm}$ & $\begin{array}{l}4 \mathrm{TI} \\
\text { tumor } \\
\text { murine } \\
\text { model }\end{array}$ & $\begin{array}{l}\text { This therapy offered a long-term immune } \\
\text { memory response, which efficiently } \\
\text { protected mice from tumor recurrence } \\
\text { and metastasis. }\end{array}$ & [94] \\
\hline $\begin{array}{l}\text { Pyropheoph- } \\
\text { orbide }\end{array}$ & $\begin{array}{l}\text { Cyclodextrin-grafted } \\
\text { hyaluronic acid (HA- } \\
\text { CD) }\end{array}$ & PD-LI & JQI & $671 \mathrm{~nm}$ & $\begin{array}{l}\text { Panc02- } \\
\text { pancreatic } \\
\text { tumor } \\
\text { model }\end{array}$ & $\begin{array}{l}\text { This study consistently verified that it } \\
\text { could elicit a potent antitumor immune } \\
\text { response in pancreatic cancer while } \\
\text { relieving immunosuppression. }\end{array}$ & [96] \\
\hline IR775 & Liposome & PD-LI & Metformin & $785 \mathrm{~nm}$ & $\begin{array}{l}\text { MB49 and } \\
\text { CT26 } \\
\text { tumor } \\
\text { murine } \\
\text { models }\end{array}$ & $\begin{array}{l}\text { The weights of collected tumors in the } \\
\text { PBS group were 8-fold heavier than that } \\
\text { in the NPs + Laser group, indicating this } \\
\text { therapy possessed excellent antitumor } \\
\text { efficacy for various types of cancers. }\end{array}$ & [97] \\
\hline
\end{tabular}




\section{IDO Inhibitor Combined with PDT}

IDO is known as an intracellular enzyme that is often overexpressed by many types of tumors. Based on tumor biological reaction, IDO catalyzes tryptophan to be catabolized, thereby facilitating $\mathrm{T}$ lymphocytes' futility in the process of tumor proliferation. In addition, IDO also promotes the activation of $\mathrm{T}$ regulatory cells (Tregs), which suppresses immune reactions in various ways to modulate the TME. ${ }^{80}$ Therefore, it is important to control the IDO expression during the process of reversing ITM. Recent preclinical researches developed multiple IDO inhibitors, which offered a promising method to provoke ICD with ITM reversion. The synergistic action of PDT and IDO inhibitors induces systemic antitumor immune responses and suppresses the proliferation of primary and distant tumors.

The developed small molecule antagonists against IDO receptors such as 1-methyl-tryptophan, ${ }^{81}$ INCB24360, ${ }^{82}$ and NLG919 ${ }^{83}$ can efficiently prevent tryptophan from catabolizing. However, these molecules showed unsatisfied antitumor efficacy due to the ineffectiveness of antigen-presenting and weak $\mathrm{T}$ cell-mediated immune responses. Recently, MOF nanoparticles loading PS Ce6 and IDO inhibitors (IDOi@TBC-Hf) could be used for the combination of immunotherapy and PDT, which was reported by Lu et al. ${ }^{84}$ Tumor-bearing mice were administrated with IDOi@TBC-Hf and light, inducing excessive ROS generation accompanied by the release of DAMPs. At the same time, NPs released an IDO inhibitor that can regulate catabolism of tryptophan, resulted in ITM revision. The combined effects of ICD triggered by PDT and IDO blockade were supposed to promote cytotoxic $\mathrm{T}$ lymphocytes migrating and infiltrating in distant untreated tumors. As the researchers assessed the abscopal efficacy of IDOi@TBC-Hf in a bilateral tumor model of CT26 and MC38 colorectal cancers, the volumes of distant tumors came to significantly shrink on day 5 . These results suggested this combined therapy provoked efficient antitumor immunity in vivo and emerged as a prospective treatment for colorectal cancers in the clinic.

Further, Liu et al developed a redox-responsive liposome by self-assembling phospholipid and PS porphyrin conjugates with IDO blockade loaded to induce ICD and reverse immunosuppressive TME. ${ }^{85}$ The liposome was prepared as shown in Figure 4A, and its structure enables NPs to have long blood circulation and improved accumulation in tumors after intravenous injection. Redox-induced PDT was supposed to prompt $\mathrm{CD}^{+} \mathrm{T}$ cells infiltrating in tumors via ICD
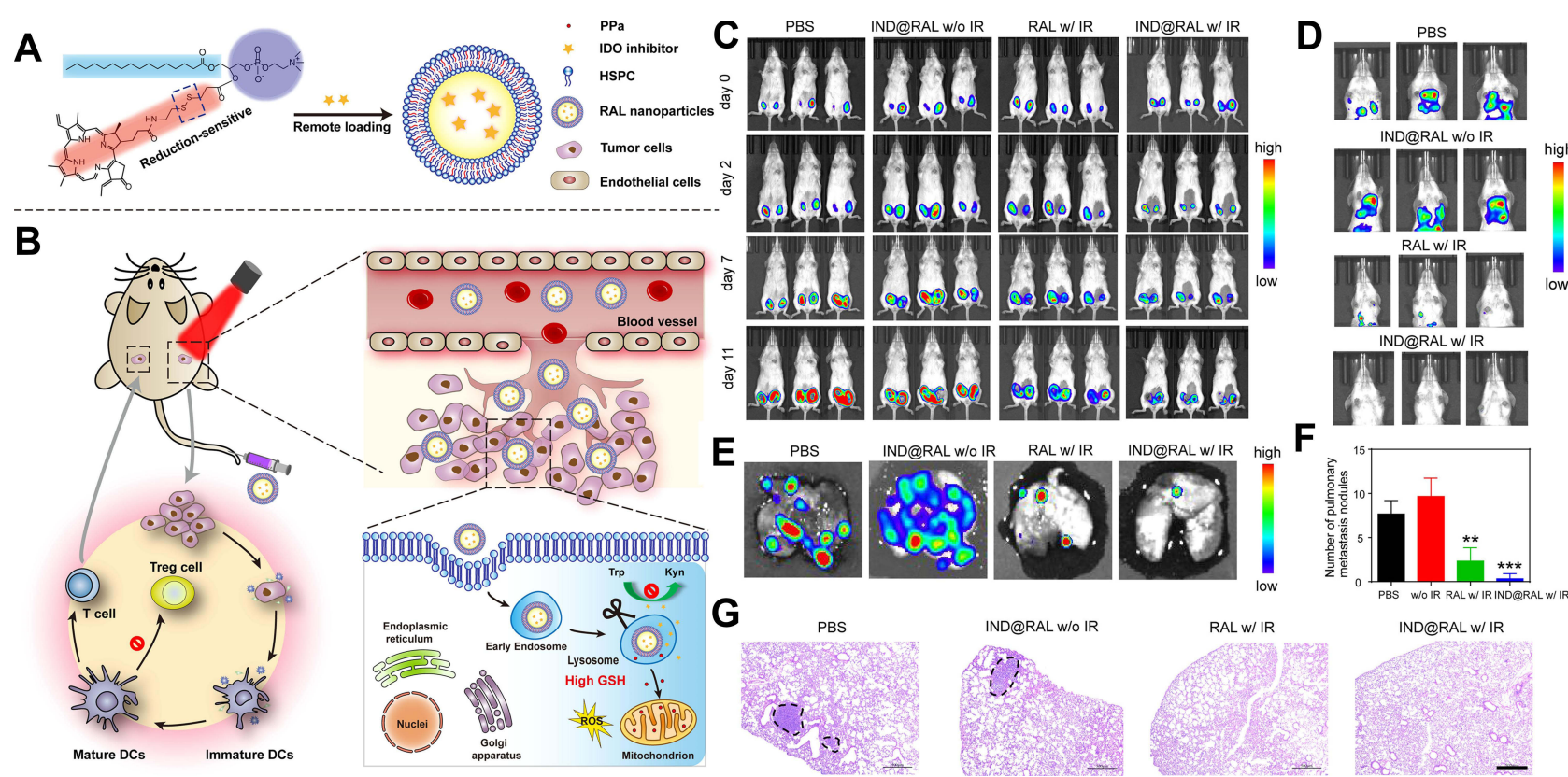

$\mathbf{F}$
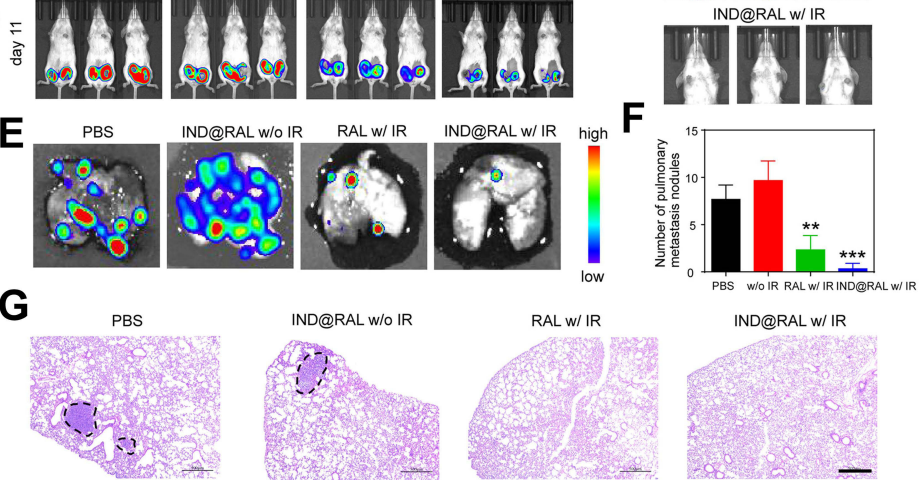

Figure 4 The anti-metastatic efficacy of porphyrin-phospholipid combined with the IDO inhibitor. (A) Schematic illustration of the preparation of functional liposomes and the mechanism of ICD pathway. (B) Antitumor mechanism of combining PDT with the IDO inhibitor in vivo. (C) Tumor growth was detected by the IVIS imaging system. (D and E) The progress of lung metastasis was observed by the IVIS imaging system. (F) The analysis of lung metastatic nodules' numbers. **P< 0.0 I, compared with PBS and IND@RAL without irradiation groups; ***P < 0.00I, compared with PBS and IND@RAL without irradiation groups. (G) H\&E stained images of lungs. Scale bar = I00 $\mu$ m.

Note: Reprinted with permission from Liu D, Chen B, Mo Y, et al. Redox-Activated Porphyrin-Based Liposome Remote-Loaded with Indoleamine 2,3-Dioxygenase (IDO) Inhibitor for Synergistic Photoimmunotherapy through Induction of Immunogenic Cell Death and Blockage of IDO Pathway. Nano Lett. 20I9; I9(I0):6964-6976. doi: I0.I02I/ acs.nanolett.9b02306. Copyright 2019 American Chemical Society. ${ }^{85}$ 
induction. As shown in Figure 4B, liposomes could accumulate in the tumor, inducing PDT efficacy, and the application of IDO inhibitor could further augment antitumor immunity. The results of antitumor metastasis experiments in a 4T1bearing mice model confirmed PDT with IDO inhibitor could significantly inhibit lung metastasis and shrink distant tumors compared to the other groups (Figure 4C-F). As shown in Figure 4G, lung metastasis was observed by H\&E staining, which demonstrated the strategy could significantly inhibit tumor metastasis.

Recently, novel self-delivery photo-immune stimulators were fabricated via the hydrophobic/electrostatic interaction of NLG919 and Ce6 by Zhao and colleagues. $^{86}$ They chose $\mathrm{Ce} 6$ as PS to generate ROS upon $660 \mathrm{~nm}$ laser, inducing DAMPs responses. NLG919 was known as a small molecule antagonist of IDO, which was applied for immunotherapy. When Ce6 and NLG919 formed NPs, it could reduce the adverse effects of drugs. Zhao groups established CT26 metastatic and 4T1 orthotopic tumor models for evaluating NPs' efficacy in treating tumor development and metastasis. The results showed the synergistic PDT and IDO inhibitor molecule had dramatical inhibition of local and distant tumor growth. Moreover, about $12.3 \%$ of Tregs were found to infiltrate in tumor sites, which was 4.7-folder lower than the PBS group, indicating a tremendous reversion of ITM.

\section{CTLA-4 Blockade Combined with PDT}

CTLA-4 is known as a transmembrane receptor that is largely expressed on $\mathrm{T}$ cell surface. Due to the specific bond with APCs via CD86 or CD80 receptors, CTLA-4 effectively suppresses the activation of cytotoxic $\mathrm{T}$ lymphocytes, causing the decrease of effector $\mathrm{T}$ cells and enhance of Tregs in cancer treatments. As anti-CTLA -4 antibodies block CTLA on Tregs, the immunosuppression induced by $\mathrm{T}$ lymphocytes would be largely reduced. ${ }^{87}$ As previously introduced, UCNP is a kind of advanced nanomaterials, which can transfer NIR light to visible luminescence, enhancing tissue penetration depth of PDT. Recently, Liu and colleagues developed a nanoplatform consisting of UCNP-cloaked with PEG, Ce6, and a small molecule R837 for curing tumors in deeper tissues. ${ }^{88}$ The light spot of the research is the combined application of PDT and anti-CTLA-4 antibodies' injection. This treatment approach produced an enhanced proportion of $\mathrm{CD}^{+} \mathrm{T}$ cells to Tregs in distant tumors, and the large amounts of secretion of proinflammatory cytokines such as IFN- $\gamma$, TNF- $\alpha$. Meanwhile, a greater proportion of $\mathrm{T}$ effector memory cells was found in the combined treated group compared to the other groups in a rechallenged mice model with the second inoculation of CT26 cells, indicating this approach had an enduring immune memory response, which protected mice from tumor recurrence.

Similarly, Lin et al applied UCNP as a NIR sensor to produce deep tissue PDT and coated UCNP with gold nanoparticles to increase the luminescence intensity, achieving real-time dynamic imaging of mitochondria. ${ }^{89}$ Besides, the application of $\alpha C$ TLA- 4 as an ICB could effectively reverse ITM and enhanced the antitumor efficacy of PDT. The anticancer efficacy experiment in vivo was taken on an orthotopic 4T1 mice model. The results indicated that the tumor sizes in the PBS, PDT alone, and PDT $+\alpha$ CTLA-4 groups were about 1173, 977, $52 \mathrm{~mm}^{3}$, indicating the combined PDT with an ICB had a promising prospect to be applied in the clinic.

In recent years, the cell membrane coated drug delivery system is arising as a novel biocompatible tumor-targeting nanomedicine. For instance, Wang et al developed novel nanobullets loaded with Ce6 utilizing mesoporous organosilica structures, which were called M-MONs@Ce6 for achieving $\mathrm{pH} /$ redox-responsive $\mathrm{Ce} 6$ release. ${ }^{90}$ In addition, they used cancer membranes to decorate NPs' surface for strengthening tumor accumulation and avoiding macrophage internalization. The results exhibited ICD provoked by PDT and magnetic hyperthermia had a synergistic effect with $\alpha$ CTLA-4 injection, which efficiently exhibited more DAMP release, lung metastasis inhibition with lower side effects on an orthotropic 4T1 $\mathrm{BALB} / \mathrm{c}$ mouse tumor model. This study meaningfully expanded the combined application of PDT, hyperthermia, and immunotherapy via the ICB pathway in the cancer metastasis research field.

\section{PD-LI/PD-I Blockade Combined with PDT}

Compare to CTLA-4, PD-1 is a widely outer membrane expressed by immune cells, such as natural killer cells, T lymphocytes, B cells, DCs, and macrophages, eliciting immune suppression effects. Cytotoxic $\mathrm{T}$ lymphocytes are blocked by PD-1 in its late stage through the specific binding to PD-L1, PD-L2 on immune or tumor cells. ${ }^{91}$ Notably. PD-L1 is overexpressed on tumor cells, which has a significant upregulation when mature DCs and activated $\mathrm{T}$ lymphocytes secreting proinflammatory cytokines. Thus, cancers have a strong immune escaping ability via the overexpression of PD-L1. ${ }^{92}$ 
Immunogenic PDT stimulates tumors to be sensitive to immune checkpoints, and the application of anti-PD-1/PDL1 antibodies can destroy primary tumors with metastasis inhibition. For instance, Duan et al reported core-shell NPs loaded with $\mathrm{Zn}$ in the core and PS pyrolipid in the shell for highly efficient PDT. ${ }^{93}$ The in vivo experiments suggested that the PDT combined with PD-L1 blockade treatment significantly inhibited lung metastasis in the triplenegative breast cancer (TNBC) murine model. Although anti-PD-L1/PD-1 antibodies are widely applied to block immune inhibitory pathways in the clinic, these antibodies remain many shortages, such as long half-life, limited tissue penetration, lack of immunogenicity, expensive production. Therefore, some researches focused on developing non-antibody-based agents to block the PD-L1/PD-1 pathway within this field. For instance, Zhang et al found a small molecule, named BMS-202, which blocked the PD-L1/PD-1 pathway that was produced by BMS. They applied the reprecipitation method to form Ce6/BMS-202 nanoparticles for combined therapy of PDT and ICB. ${ }^{94}$ It was noted that Ce6/BMS-202 nanoparticles with laser irradiation led to a $91.1 \%$ tumor inhibition rate at the endpoint. In comparison, this value was $92.6 \%$ for $\mathrm{Ce} 6$ nanoparticles with laser irradiation plus $\alpha$-PD-L1, demonstrating a difference of only $1.5 \%$. The results demonstrated that BMS-202 nanoparticles possessed similar efficacy of anti-PD-L1 antibodies for eradicating local and abscopal tumors, offering long-term immune memory responses to prevent cancer recurrence in a TNBC murine model.

Oxygen is the essential element in producing PDT efficacy, however, tumor hypoxia microenvironment strictly limited ROS generation. ${ }^{95}$ In addition, tumor hypoxia further prompts the glycolysis process, causing excessive production of lactate and severe ITM. Therefore, to solve this problem, Sun and colleagues developed supramolecular NPs co-delivering a PS and a prodrug named JQ1 for PDT combining immunotherapy of pancreatic cancer. $^{96}$ In addition, JQ1 acted as a bromodomain-containing protein 4 inhibitor (BRD4i), which inhibited glycolysis and prevented the transcription of c-Myc under tumor hypoxia microenvironment. More importantly, JQ1 could reverse PDT-triggered immune tolerance by significantly down-regulated tumor cells' expression of PD-L1. As shown in Figure 5A, the nanoparticles were synthesized by JQ1, PS pyropheophorbide

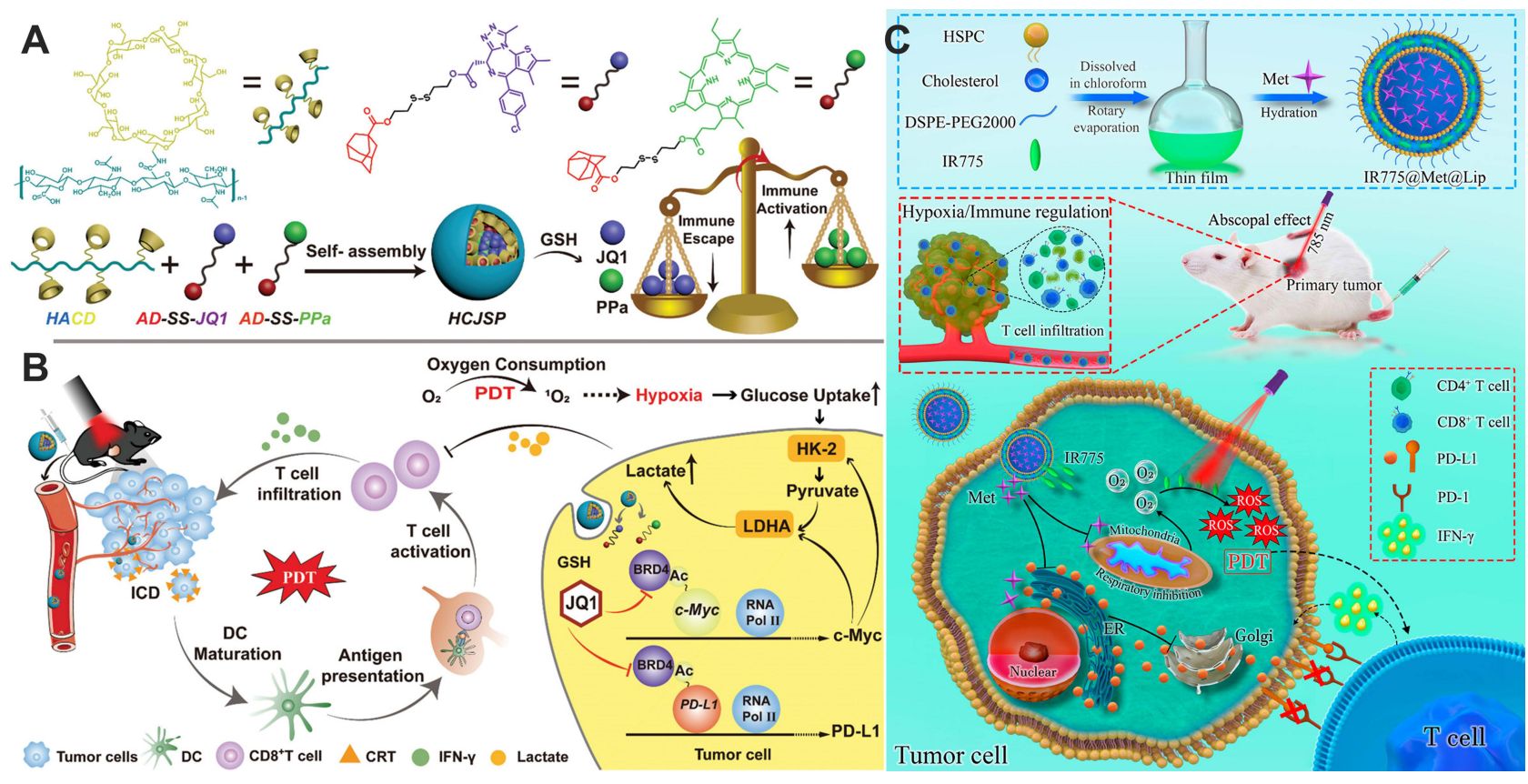

Figure 5 Different PD-LI/PD-I blockade strategy combined with PDT to better cure cancer. (A) Schematic description of NPs fabricated by the electronic interaction of AD-SS-JQI, HA-CD, and AD-SS-PPa. (B) The expected mechanism of NPs triggered a combination of PDT with immunotherapy for pancreatic cancers. Reproduced from Sun F, Zhu Q, Li T, et al. Regulating Glucose Metabolism with Prodrug Nanoparticles for Promoting Photoimmunotherapy of Pancreatic Cancer. Adv Sci. 202 I;8(4):2002746. doi:10.1002/advs.202002746. ${ }^{96}$ (C) Schematic illustration of the preparation of IR775@Met@Lip and the mechanism of enhanced photodynamic immunotherapy mediated by reversing hypoxia and decreasing PD-LI expression in vivo. Reprinted with permission from Xiong W, Qi L, Jiang N, et al. Metformin Liposome-Mediated PD-LI Downregulation for Amplifying the Photodynamic Immunotherapy Efficacy. ACS Appl Mater Interfaces. 2021;13(7):8026-804I. doi:10.1021/acsami.0c2 I743. Copyright 202I American Chemical Society. ${ }^{97}$ 
a (PPa), and cyclodextrin-grafted hyaluronic acid (HACD)'s complexation. Among all ingredients, HA enabled NPs own active tumor-targeting capability via its specific binding with CD44 that overexpressed on tumor cells. As shown in Figure 5B, PPa-induced PDT improved tumor immunogenicity with an enhanced proportion of active $\mathrm{T}$ cells infiltrating intratumorally. Besides, JQ1 combated immune suppression triggered by PD-L1, preventing glycolysis and c-Myc expression in pancreatic cancer. Although pancreatic cancer possesses major difficulties in immunotherapy, including limited $\mathrm{T}$ cell infiltration, lower immunogenicity, poor responses to ICB, this combination therapy successfully enhanced ICD-induced photoimmunotherapy via evoking cytotoxic $\mathrm{T}$ cell migration and combating immune tolerance in PD-L1/PD-1 pathway. The results showed the nanocomplex with laser treatment had a 10.2-fold higher $\mathrm{CD}^{+} \mathrm{T}$ cells to Tregs ratio than that of the PBS group and the frequency of IFN$\gamma^{+}$effector T cells increased to 2.0 -fold in a pancreatic cancer murine model.

Similarly, to reverse tumor hypoxia and ITM, Xiong and his group reported the fabrication of clinically applied liposomes loaded with PS IR775 and metformin (Met) named as IR775@Met@Lip. ${ }^{97}$ Schematic illustration of the nanoparticle preparation and work mechanism in vivo was exhibited in Figure 5C. The nanoplatform delivered oxygen to reverse tumor hypoxia, promoting the generation of ROS. In addition, the loaded Met is an oral drug for regulating blood glucose in type II diabetes, which is recently found to own the capability of down-regulating PD-L1 expression on cancer membranes through pAMPK-regulated ER-related protein degradation. ${ }^{98}$ Thus, they used Met to reduce the expression of PD-L1 for achieving PDT combined immunotherapy. The outstanding advantages of this treatment including tumor hypoxia revision, alleviation of $\mathrm{T}$ lymphocyte exhaustion, leading to brilliant antimetastatic effects in both colon and bladder cancer models.

\section{PDT Combined with Other Immune Checkpoint Inhibitors or Agonists}

With the great success of anti-CTLA-4 and anti-PD-1/PD-

L1 therapeutics in cancer immunotherapy across many cancer types, tumor necrosis factor receptor superfamily members have been recognized as ideal targets to provide costimulatory signals in combination with immune checkpoint blocking antibodies. Among these is OX40 (CD134), a co-stimulatory molecule expressed by activated immune cells, which is considered as the next generation of the immune therapeutic target. Engagement of the OX40 receptor on T-cells, applying OX40 agonists, directly promotes an increase in survival of different effector T-cell subsets and counteract Tregs functions. ${ }^{99-101}$ Recently, several anti-OX40 agonistic monoclonal antibodies, pogalizumab as the most advanced, have entered early phase clinical trials. ${ }^{102}$ However, the efficacy of anti-OX40 agonists against bulk solid tumors or metastases is less impressive. Hence, different modalities of treatment combinations have been attempted to overcome resistance to anti-OX40 molecules. In order to strengthen anti-OX40 therapy, the combination of anti-OX40 with regular anti-cancer treatment, such as surgery, ${ }^{103}$ radiotherapy, ${ }^{104}$ chemotherapy ${ }^{105}$ has been investigated in preclinical research. Recently, Ricardo Alvim and colleagues reported the combined OX40 agonist and PD-1 inhibitor immunotherapy improves the efficacy of vascular targeted PDT in a urothelial tumor model. ${ }^{106}$ In mice allografted with MB-49 UTUC cells, they found that the combination of PDT with both PD-1 inhibitor and OX40 agonist inhibited tumor growth and metastasis and prolonged survival to a much greater degree than PDT with either immunotherapeutic individually. The results suggested that PDT synergizes with PD-1 blockade and OX40 agonist to promote strong antitumor immune responses, yielding therapeutic efficacy in an animal model of urothelial cancer.

Co-inhibitory receptors, such as CTLA-4 and PD-1, have an important role in cancer immunotherapy. Unfortunately, many patients still fail to respond to therapies that target CTLA-4 and PD-1. The next wave of co-inhibitory receptor targets that are being explored in clinical trials includes lymphocyte activation gene-3 (Lag-3), T cell immunoglobulin-3 (TIM-3), and T cell immunoglobulin and ITIM domain protein (TIGIT). ${ }^{107}$ These receptors, although they belong to the same class of receptors as PD-1 and CTLA-4, exhibit unique functions, especially at tissue sites where they regulate distinct aspects of immunity. It was reported that TIM-3 expression by intratumoral $\mathrm{CD}_{103}{ }^{+} \mathrm{DCs}$ regulates chemokine expression during paclitaxel treatment. And the application of anti-TIM-3 antibody during chemotherapy led to enhanced granzyme $\mathrm{B}$ expression by $\mathrm{CD} 8^{+} \mathrm{T}$ cells and an immune-mediated response. ${ }^{108}$ Jennifer and colleagues first reported the preclinical investigation on the effects of dual PD-1 and TIM-3 blockade with radiation in human glioblastoma multiforme. ${ }^{109}$ Triple therapy resulted in $100 \%$ overall survival $(\mathrm{P}<0.05)$, which was a significant improvement compared with other arms. Although the combination of 
TIM-3 with PDT has not been investigated, the above results indicated this combination therapy holds great potential in clinical application with durable clinical benefits.

\section{Strategies of Increasing Tumor Immunogenicity}

Despite PDT-triggered antitumor immunity via the ICD process, different cancers have different levels of antitumor immune responses with PDT treatment, which may account for a heterogeneous characteristic of cancers. Different cancers own various immunogenicity which decides less or more T cell infiltration. And we also call this phenomenon "hot tumors" compared to "cold tumors". ${ }^{110}$ Thus, recent researches raised a novel strategy via the combination of PDT with certain agents which could increase tumor immunogenicity and promote immune responses. Besides, as PDT produces cytotoxic ROS and damages local tumors directly, these agents can act as adjuvants to evoke strong and enduring immune responses combating metastatic cancers. These certain agents include immunoadjuvants and DC-based vaccines, which increased tumor immunogenicity with PDT-induced ICD.

\section{PDT Combined with Immunoadjuvants}

To amplify the PDT-induced ICD, immunoadjuvants exist as the main agents that contribute to prompting antitumor immune responses. Some immunoadjuvants have been widely used for tumor inhibition in preclinical researches, such as ovalbumin (OVA), granulocyte-macrophage colonystimulating factor (GM-CSF), and oligonucleotides containing cytosine-guanine motifs $(\mathrm{CpG}) .{ }^{111}$ As previously mentioned, CRT is a $\mathrm{Ca}^{2+}$ interacting protein, which is released from apoptotic cells as an "eat me" signal. ${ }^{112}$ The immunoadjuvants such as CpG and OVA have similar effects in stimulating immune systems, which suggests the reasonability of combining ICD-boosted therapies with immunoadjuvants. For example, Xia and colleagues applied PDT combined with $\mathrm{CpG}$ in a breast cancer murine model. ${ }^{113}$ First, $\mathrm{CpG}$ activated DCs' maturation through toll-like receptor 9, contributing to synergistic effects with PDT induced ICD in vitro. Meanwhile, after treating with PDT, administration of $\mathrm{CpG}$ inhibited tumor growth and metastasis, improved mice survival compared with other single treatments in a 4T1 murine mice model. Chen et al reported the application of a series of immunoadjuvants to enhance PDT efficacy via activation of immune responses. ${ }^{114}$ They concluded that N-dihydrogalactochitosan (GC) owned the best immune activation ability compared with other tested immunoadjuvants. Korbelik et al reported the combined application of GC with PDT in a squamous cell cancer murine model. ${ }^{115}$ PDT with assistant GC treatment significantly decreased the numbers of myeloid-derived suppressor cells (MDSCs), promoting therapeutic benefits. To investigate the function mechanism of CG plus PDT, they used a caspase-1 blockade to confirm this enzyme's main role in repairing membrane lipids. Thus, GC is supposed as the suitable immunoadjuvant for combing PDT treatment, which also fits other tumor therapies.

As known to all, Toll-like receptors (TLR) are effective in identifying antigens and subsequently evoking DCs maturation. The surface of DCs is expressed with various TLR receptors, including TLR-9, TLR-4, TLR-3, TLR-2, and TLR-13. PDT can increase the secretion of HMGB1, which can interact with TLR on DCs, resulting in activation and infiltration of cytotoxic $\mathrm{T}$ lymphocytes. Thus, the synchronous application of TLR agonists and PDT can efficiently stimulate immune responses. For instance, Hwang et al reported the application of PDT and flagellinadjuvanted tumor-specific peptide vaccination (FlaB-Vax) supposed as a TLR-5 agonist in a B16-F10 bearing mice model. ${ }^{116}$ The results indicated PDT with Flab-Vax could improve mature DCs' accumulation in lymph nodes for antigen-presenting and the proportion of $\mathrm{CD}^{+}$cells in $\mathrm{T}$ lymphocytes. Recently, Ni and colleagues found the application of nanoscale MOFs co-delivered TLR-7 agonists imiquimod (IMD) and $\alpha \mathrm{CD} 47$ to mediate the function of macrophages and arrange immunotherapy, as shown in Figure 6A. ${ }^{117}$ IMD modulated macrophages from immunosuppressive M2 to immune-stimulatory M1, while antiCD47 antibodies blocked CD47 expressed on the tumor cell surface, strengthening antitumor immunity. This nanomaterials-based co-delivery of IMD and $\alpha \mathrm{CD} 47$ served as a general strategy to tune the TME for synergistic combination with immunotherapies. Significant increases of antigenspecific IFN- $\gamma$ producing T cells were observed in IMD plus $\alpha \mathrm{CD} 47$ with PDT group $\left(61.6 \pm 28.6 / 10^{6}\right.$ cells $)$ compared to $8.0 \pm 9.3 / 10^{6}$ cells for PBS group, suggesting induction of strong tumor-specific adaptive immunity. The results in Figure 6B-D sufficiently confirmed that this strategy provided a nanoplatform co-loading immunoadjuvants and ICB with X-ray induced RT and PDT, for macrophages repolarization and a long-term immunotherapy. Other TLR agonists such as mycobacterium cell wall extract (MCWE) can also be used to combine with PDT using various PS, resulting in eradicating both local and distant tumors. ${ }^{118}$ 

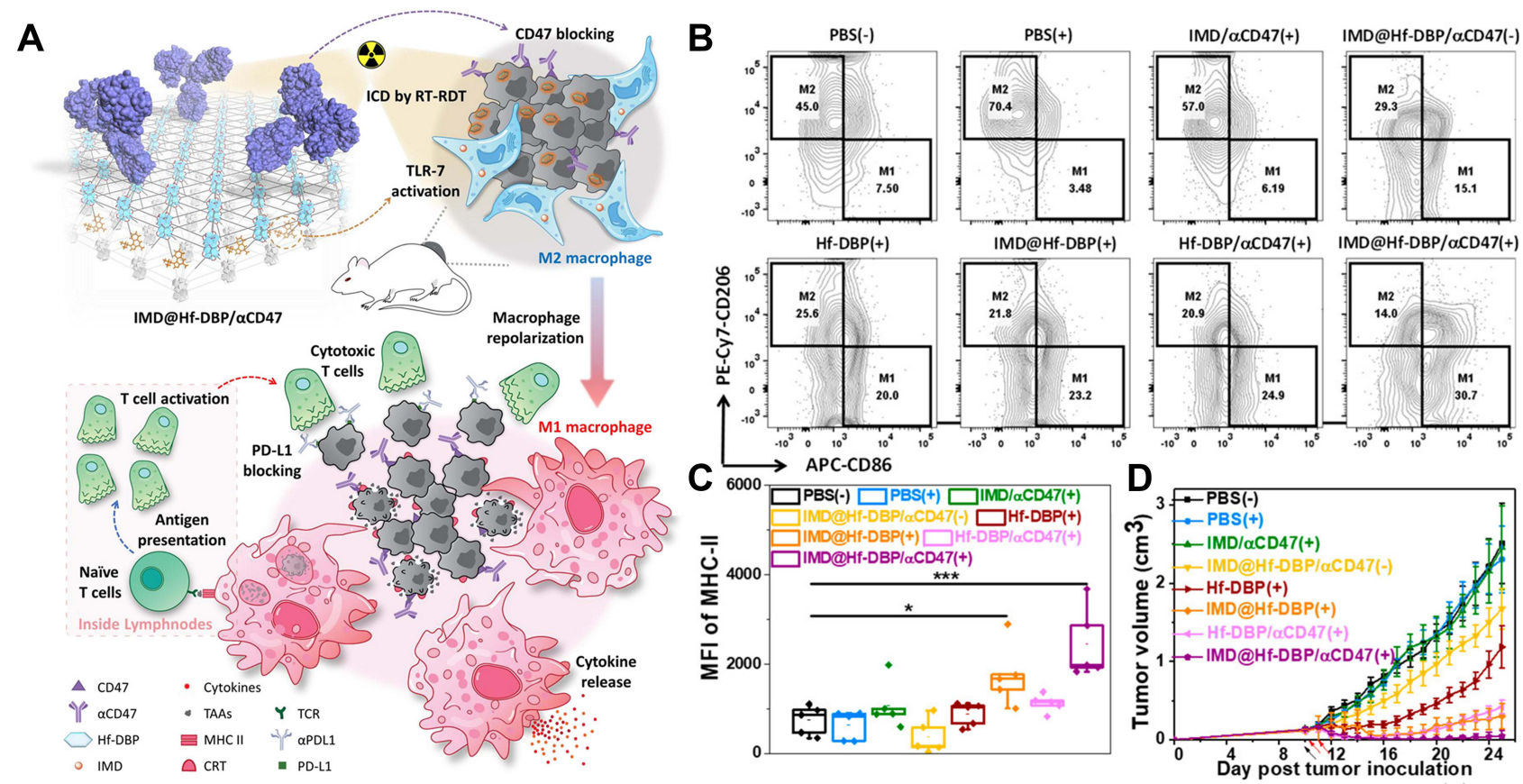

Figure 6 Illustration of IMD@Hf-DBP/ $\alpha$ CD47 plus radiotherapy and PDT. (A) The design of RT-PDT induced ICD combined with the immunoadjuvant and ICB to systemically eradicate tumors. (B) Flow cytometry analysis of mature DCs. (C) Fluorescence intensity of MHC-II. $* P<0.05$, and $* * * P<0.005$ from control. (D) Tumor growth curves with various treatments in CT26-bearing murine model. Black and red arrows refer to intratumoral injection and X-ray irradiation, respectively. Note: Reprinted with permission from Ni K, Luo T, Culbert A, Kaufmann M, Jiang X, Lin W. Nanoscale Metal-Organic Framework Co-delivers TLR-7 Agonists and AntiCD47 Antibodies to Modulate Macrophages and Orchestrate Cancer Immunotherapy. J Am Chem Soc. 2020;142(29): I2579-12584. doi: 0 .102 I/jacs.0c05039. Copyright 2020 American Chemical Society. ${ }^{17}$

\section{PDT-DC Vaccines}

With the advancement in specific molecular recognition of peptide sequences, more and more researches focused on establishing antitumor vaccines with a rational design. Numerous kinds of vaccines have been investigated as well as PDT-induced DC vaccines. There are more than 200 accomplished clinical experiments for exploring DC-based vaccines targeted for cancer therapies. ${ }^{119}$ Previous reports have confirmed that PDT induced immature DCs to be matured and spontaneous migration with increased proinflammatory cytokine release. It has been convinced that cancer cells with PDT treatment could be used as an adjuvant for DC-based vaccines (PDT-DC vaccines), ${ }^{120}$ which trigger T cell-mediated immune responses in comparison with untreated cancer cells. ${ }^{121}$

For instance, using B16 melanoma and CT26 colorectal carcinoma murine models, Saji et al demonstrated that this treatment improved completed cancer-cured proportions in vivo trials and lengthened survival of remained mice. ${ }^{122}$ Notably, the abscopal metastasis was significantly inhibited for a long time under the treatment, indicating the amplified systemic antitumor immunity. Molecularly defined therapeutic peptide vaccination has been successfully combined with Ce6-based PDT in murine models and convincingly showed synergistic clearance of primary tumors. Recently, a Ce6-triggered PDT combined with therapeutic peptide vaccination was successfully established and applied to inhibit tumor growth and eradicate tumor metastasis in TC-1 tumor-bearing mice. Moreover, significantly increased $\mathrm{CD} 8^{+} \mathrm{T}$ cell infiltrating was found in the secondary tumors. ${ }^{123}$ Yang and colleagues established a prophylactic vaccine via PDT-treated tumor lysate. Specifically, they used DTPP-regulated PDT in vitro and found a boosted ratio of $\mathrm{CD}^{+} / \mathrm{CD}^{+}$cells, elevated IL-1 and IFN- $\gamma$ secretions in serum, and increased natural killer (NK) cell proportions. ${ }^{124}$ These PDT-DC vaccines are more effective in eradicating tumors and have stronger abilities to trigger antitumor immunity, which can also enhance the $\mathrm{T}$ lymphocyte response. Thus, the PDT-DC vaccine was supposed as a relatively ideal combination therapy strategy.

\section{PDT Combined with Other Approaches}

Some special enzymes or peptides which can increase tumor immunogenicity during PDT-induced ICD, are widely applied to form PDT-combined therapy. For instance, recent researches showed that acid ceramidase, known as an important enzyme, which can regulate the levels of sphingosine and ceramide, is 
very vital in the cell apoptotic process. The overexpressed acid ceramidase was widely discovered on cancer cells, inducing apoptotic resistance on them. ${ }^{125}$ Therefore. LCL521 known as an acid ceramidase inhibitor is worthy of detailed investigation.

For instance, Korbelik et al applied LCL521 in combination with PDT for tumor therapy. The results demonstrated that this therapy could remarkedly suppress Tregs and MDSCs proliferation in lymph nodes compared to a single PDT treatment which was detected by flow cytometry. ${ }^{126}$ However, the suppression efficacy was not found in spleens, which suggested that the use of LCL521 enabled PDT to achieve its complete immune-activating ability to recruit cytotoxic $\mathrm{CD}^{+}$ $\mathrm{T}$ cells and macrophages. In addition, bremachlorin-induced PDT combined with synthetic long peptides (SLP) that owned epitopes from tumor antigens was established for treating RMA and TC-1 cancer murine models, which was reported by JW et al. ${ }^{123}$ This treatment aroused an obvious inhibition of tumor growth with about one-third of mice completely cured. Moreover, the cured mice survived without cancer recurrence for a long time. Besides, the activated immune responses successfully eradicated the abscopal secondary tumors. Although PDT applied alone owned antitumor immunity via eliciting ICD, the immune responses would be dramatically boosted through the combined application of SLP. Thus, SLP with PDT is emerging as a promising approach for treating intractable cancers.

\section{Summary and Future Perspectives}

PDT has the obvious advantages of a small injury, low side effects, precise treatment, and simple operation among all the antitumor therapies. However, PDT is limited by the lack of tumor targeting, depth of treatment penetration, and hypoxia TME. Recently, with the development of nanotechnology, nanomaterials-based PDT overcomes the above shortages and expands the application of PDT in preclinical research. Nanomaterials-based PDT shows specific tumor targeting and accumulating ability, that provides deeper treatment depth with reduction of side effects, and achieves much ideal efficacy in tumor therapeutic applications than conventional PDT. More importantly, PDT induced ICD, which activates $\mathrm{T}$ cellmediated immune responses through danger signaling mechanisms induced by DAMPs. However, these antitumor immune responses are not strong enough to eradicate tumor residues for T cell responses induced by ICD are widely limited in vivo. To enhance the efficacy of ICD-based PDT, the nanomaterials-based drug delivery system was designed with several drugs or agents loaded, which amplified antitumor immune responses. The combined application of PDT and
ICD-based tumor immunotherapy has a great potential to improve systematic antitumor immunity with an enduring immune memory impact by simultaneously increasing tumor immunogenicity and relieving immunoregulatory suppression.

Despite great advances that have been made in these nanomaterials-based PDT with amplified ICD treatments, there are still several challenges in their clinical application. Firstly, many new traits caused by nanomaterials-based PDT combined with immunotherapy strategy need to be carefully investigated. For example, the fabricated nanomedicine needs to receive investigations about its properties of absorption, distribution, metabolism, excretion, and toxicity in vivo. Secondly, the developed method of controlling NPs' structure and quality with a simple design of nanomedicine is critical to the clinical translation. However, the nanoparticles designed by the most current studies are too complex to apply in the clinic. Thirdly, more specific researches about the mechanism of PDT-immunotherapy eliciting ICD should be exhibited for basic research and clinical translation.

\section{Acknowledgment}

This work is supported by China Postdoctoral Science Foundation funded project (2020M681902), Postdoctoral Foundation of Zhejiang Province (ZJ2020034), which provided by the Fundamental Research Funds for the Central Universities (2021QNA7020).

\section{Author Contributions}

All authors made substantial contributions to conception and design, acquisition of data, or analysis and interpretation of data; took part in drafting the article or revising it critically for important intellectual content; agreed to submit to the current journal; gave final approval of the version to be published; and agree to be accountable for all aspects of the work.

\section{Disclosure}

The authors declare no competing financial or other interests.

\section{References}

1. Bray F, Ferlay J, Soerjomataram I, Siegel RL, Torre LA, Jemal A. Global cancer statistics 2018: GLOBOCAN estimates of incidence and mortality worldwide for 36 cancers in 185 countries. CA Cancer J Clin. 2018;68(6):394-424. doi:10.3322/caac.21492

2. Li X, Lovell JF, Yoon J, Chen X. Clinical development and potential of photothermal and photodynamic therapies for cancer. Nat Rev Clin Oncol. 2020;17(11):657-674. doi:10.1038/s41571-020-0410-2

3. Li Y, Li X, Zhou F, et al. Nanotechnology-based photoimmunological therapies for cancer. Cancer Lett. 2019;442:429-438. doi:10.1016/j. canlet.2018.10.044 
4. Abrahamse H, Hamblin MR. New photosensitizers for photodynamic therapy. Biochem J. 2016;473(4):347-364. doi:10.1042/ bj20150942

5. Idris NM, Jayakumar MK, Bansal A, Zhang Y. Upconversion nanoparticles as versatile light nanotransducers for photoactivation applications. Chem Soc Rev. 2015;44(6):1449-1478. doi:10.1039/c4cs00158c

6. Zhou Z, Zhang B, Wang H, Yuan A, Hu Y, Wu J. Two-stage oxygen delivery for enhanced radiotherapy by perfluorocarbon nanoparticles. Theranostics. 2018;8(18):4898-4911. doi:10.7150/ thno. 27598

7. Gao A, Chen B, Gao J, et al. Sheddable Prodrug Vesicles Combating Adaptive Immune Resistance for Improved Photodynamic Immunotherapy of Cancer. Nano Lett. 2020;20 (1):353-362. doi:10.1021/acs.nanolett.9b04012

8. Zhou Z, Zhang B, Zai W, et al. Perfluorocarbon nanoparticle-mediated platelet inhibition promotes intratumoral infiltration of $\mathrm{T}$ cells and boosts immunotherapy. Proc Natl Acad Sci U S A. 2019;116(24):11972-11977. doi:10.1073/ pnas. 1901987116

9. Wang D, Wang T, Liu J, et al. Acid-Activatable Versatile Micelleplexes for PD-L1 Blockade-Enhanced Cancer Photodynamic Immunotherapy. Nano Lett. 2016;16 (9):5503-5513. doi:10.1021/acs.nanolett.6b01994

10. Yeung HY, Lo PC, Ng DK, Fong WP. Anti-tumor immunity of BAM-SiPc-mediated vascular photodynamic therapy in a BALB/ c mouse model. Cell Mol Immunol. 2017;14(2):223-234. doi:10.1038/cmi.2015.84

11. Nam J, Son S, Park KS, Zou W, Shea LD, Moon JJ. Cancer nanomedicine for combination cancer immunotherapy. Nat Rev Mater. 2019;4(6):398-414. doi:10.1038/s41578-019-0108-1

12. Dolmans DE, Fukumura D, Jain RK. Photodynamic therapy for cancer. Nat Rev Cancer. 2003;3(5):380-387. doi:10.1038/nrc1071

13. Calixto GM, Bernegossi J, de Freitas LM, Fontana CR Chorilli M. Nanotechnology-Based Drug Delivery Systems for Photodynamic Therapy of Cancer: a Review. Molecules. 2016;21 (3):342. doi: $10.3390 /$ molecules 21030342

14. Li X, Kwon N, Guo T, Liu Z, Yoon J. Innovative Strategies for Hypoxic-Tumor Photodynamic Therapy. Angew Chem Int Ed Engl. 2018;57(36):11522-11531. doi:10.1002/anie.201805138

15. Kwiatkowski S, Knap B, Przystupski D, et al. Photodynamic therapy - mechanisms, photosensitizers and combinations. Biomed Pharmacother. 2018;106:1098-1107. doi:10.1016/j. biopha.2018.07.049

16. Agostinis P, Berg K, Cengel KA, et al. Photodynamic therapy of cancer: an update. CA Cancer J Clin. 2011;61(4):250-281. doi:10.3322/caac. 20114

17. Yang $\mathrm{G}, \mathrm{Xu} \mathrm{L}$, Chao $\mathrm{Y}$, et al. Hollow $\mathrm{MnO}_{2}$ as a tumor-microenvironment-responsive biodegradable nano-platform for combination therapy favoring antitumor immune responses. Nat Commun. 2017;8(1):902. doi:10.1038/ s41467-017-01050-0

18. Zhou Z, Zhang B, Wang S, et al. Perfluorocarbon Nanoparticles Mediated Platelet Blocking Disrupt Vascular Barriers to Improve the Efficacy of Oxygen-Sensitive Antitumor Drugs. Small. 2018;14(45):e1801694. doi:10.1002/smll.201801694

19. Chen Q, Wang X, Wang C, Feng L, Li Y, Liu Z. Drug-Induced Self-Assembly of Modified Albumins as Nano-theranostics for Tumor-Targeted Combination Therapy. ACS Nano. 2015;9 (5):5223-5233. doi:10.1021/acsnano.5b00640

20. Chen G, Jaskula-Sztul R, Esquibel CR, et al. Neuroendocrine Tumor-Targeted Upconversion Nanoparticle-Based Micelles for Simultaneous NIR-Controlled Combination Chemotherapy and Photodynamic Therapy, and Fluorescence Imaging. Adv Funct Mater. 2017;27:8. doi:10.1002/adfm.201604671
21. Yang G, Tian J, Chen C, et al. An oxygen self-sufficient NIR-responsive nanosystem for enhanced PDT and chemotherapy against hypoxic tumors. Chem Sci. 2019;10(22):5766-5772. doi:10.1039/c9sc00985j

22. Hu J, Tang Y, Elmenoufy AH, Xu H, Cheng Z, Yang X. Nanocomposite-Based Photodynamic Therapy Strategies for Deep Tumor Treatment. Small. 2015;11(44):5860-5887. doi:10.1002/smll.201501923

23. Mishchenko T, Mitroshina E, Balalaeva I, Krysko O, Vedunova M, Krysko DV. An emerging role for nanomaterials in increasing immunogenicity of cancer cell death. Biochim Biophys Acta Rev Cancer. 2019;1871(1):99-108. doi:10.1016/j. bbcan.2018.11.004

24. Garg AD, Krysko DV, Verfaillie T, et al. A novel pathway combining calreticulin exposure and ATP secretion in immunogenic cancer cell death. EMBO j. 2012;31(5):1062-1079. doi:10.1038/emboj.2011.497

25. Vanden Berghe T, Kalai M, Denecker G, Meeus A, Saelens X, Vandenabeele P. Necrosis is associated with IL-6 production but apoptosis is not. Cell Signal. 2006;18(3):328-335. doi:10.1016/j. cellsig.2005.05.003

26. Xie Y, Hou W, Song X, et al. Ferroptosis: process and function. Cell Death Differ. 2016;23(3):369-379. doi:10.1038/ cdd.2015.158

27. Zhang F, Li F, Lu GH, et al. Engineering Magnetosomes for Ferroptosis/Immunomodulation Synergism in Cancer. ACS Nano. 2019;13(5):5662-5673. doi:10.1021/acsnano.9b00892

28. Wachowska M, Muchowicz A, Demkow U. Immunological aspects of antitumor photodynamic therapy outcome. Cent Eur J Immunol. 2015;40(4):481-485. doi:10.5114/ceji.2015.56974

29. Bianchi ME. DAMPs, PAMPs and alarmins: all we need to know about danger. J Leukoc Biol. 2007;81(1):1-5. doi:10.1189/ jlb.0306164

30. Garg AD, Dudek AM, Agostinis P. Cancer immunogenicity, danger signals, and DAMPs: what, when, and how? Biofactors. 2013;39(4):355-367. doi:10.1002/biof.1125

31. Tesniere A, Panaretakis T, Kepp O, et al. Molecular characteristics of immunogenic cancer cell death. Cell Death Differ. 2008;15 (1):3-12. doi:10.1038/sj.cdd.4402269

32. Alzeibak R, Mishchenko TA, Shilyagina NY, Balalaeva IV, Vedunova MV, Krysko DV. Targeting immunogenic cancer cell death by photodynamic therapy: past, present and future. J Immunother Cancer. 2021;9(1). doi:10.1136/jitc-2020-001926

33. Casares N, Pequignot MO, Tesniere A, et al. Caspase-dependent immunogenicity of doxorubicin-induced tumor cell death. J Exp Med. 2005;202(12):1691-1701. doi:10.1084/jem.20050915

34. Humeau J, Lévesque S, Kroemer G, Pol JG. Gold Standard Assessment of Immunogenic Cell Death in Oncological Mouse Models. Methods Mol Biol. 2019;1884:297-315. doi:10.1007/ 978-1-4939-8885-3 2

35. Canti G, Lattuada D, Nicolin A, Taroni P, Valentini G, Cubeddu R. Antitumor immunity induced by photodynamic therapy with aluminum disulfonated phthalocyanines and laser light. Anticancer Drugs. 1994;5(4):443-447. doi:10.1097/00001813199408000-00009

36. Shimizu K, Iyoda T, Okada M, Yamasaki S, Fujii SI. Immune suppression and reversal of the suppressive tumor microenvironment. Int Immunol. 2018;30(10):445-454. doi:10.1093/intimm/dxy042

37. Adkins I, Fucikova J, Garg AD, Agostinis P, Špíšek R. Physical modalities inducing immunogenic tumor cell death for cancer immunotherapy. Oncoimmunology. 2014;3(12):e968434. doi:10.4161/21624011.2014.968434

38. Tesniere A, Schlemmer F, Boige V, et al. Immunogenic death of colon cancer cells treated with oxaliplatin. Oncogene. 2010;29 (4):482-491. doi:10.1038/onc.2009.356 
39. Khdair A, Chen D, Patil Y, et al. Nanoparticle-mediated combination chemotherapy and photodynamic therapy overcomes tumor drug resistance. $J$ Control Release. 2010;141(2):137-144. doi:10.1016/j.jconrel.2009.09.004

40. He C, Duan X, Guo N, et al. Core-shell nanoscale coordination polymers combine chemotherapy and photodynamic therapy to potentiate checkpoint blockade cancer immunotherapy. Nat Commun. 2016;7:12499. doi:10.1038/ncomms12499

41. Ni K, Aung T, Li S, Fatuzzo N, Liang X, Lin W. Nanoscale Metal-Organic Framework Mediates Radical Therapy to Enhance Cancer Immunotherapy. Chem. 2019;5(7):1892-1913. doi:10.1016/j.chempr.2019.05.013

42. Chen Q, Liu L, Lu Y, et al. Tumor Microenvironment-Triggered Aggregated Magnetic Nanoparticles for Reinforced Image-Guided Immunogenic Chemotherapy. Adv Sci. 2019;6 (6):1802134. doi:10.1002/advs.201802134

43. Kuai R, Yuan W, Son S, et al. Elimination of established tumors with nanodisc-based combination chemoimmunotherapy. Sci Adv. 2018;4(4):eaao1736. doi:10.1126/sciadv.aao1736

44. Shi C, Li M, Zhang Z, et al. Catalase-based liposomal for reversing immunosuppressive tumor microenvironment and enhanced cancer chemo-photodynamic therapy. Biomaterials. 2020;233:119755. doi:10.1016/j.biomaterials.2020.119755

45. Jin F, Qi J, Zhu M, et al. NIR-Triggered Sequentially Responsive Nanocarriers Amplified Cascade Synergistic Effect of Chemo-Photodynamic Therapy with Inspired Antitumor Immunity. ACS Appl Mater Interfaces. 2020;12 (29):32372-32387. doi:10.1021/acsami.0c07503

46. Liu S, Doughty A, West C, Tang Z, Zhou F, Chen WR. Determination of temperature distribution in tissue for interstitial cancer photothermal therapy. Int J Hyperthermia. 2018;34 (6):756-763. doi:10.1080/02656736.2017.1370136

47. Sweeney EE, Cano-Mejia J, Fernandes R. Photothermal Therapy Generates a Thermal Window of Immunogenic Cell Death in Neuroblastoma. Small. 2018;14(20):e1800678. doi:10.1002/ smll.201800678

48. Dewhirst MW, Lee CT, Ashcraft KA. The future of biology in driving the field of hyperthermia. Int J Hyperthermia. 2016;32 (1):4-13. doi:10.3109/02656736.2015.1091093

49. Yi X, Yang $\mathrm{K}$, Liang $\mathrm{C}$, et al. Imaging-Guided Combined Photothermal and Radiotherapy to Treat Subcutaneous and Metastatic Tumors Using Iodine-131-Doped Copper Sulfide Nanoparticles. Adv Funct Mater. 2015;25(29):4689-4699. doi:10.1002/adfm.201502003

50. Chu C, Lin H, Liu H, et al. Tumor Microenvironment-Triggered Supramolecular System as an In Situ Nanotheranostic Generator for Cancer Phototherapy. Adv Mater. 2017;29:23. doi:10.1002/ adma.201605928

51. Wang M, Rao J, Wang M, et al. Cancer photo-immunotherapy: from bench to bedside. Theranostics. 2021;11(5):2218-2231. doi:10.7150/thno. 53056

52. Wang M, Song J, Zhou F, et al. NIR-Triggered Phototherapy and Immunotherapy via an Antigen-Capturing Nanoplatform for Metastatic Cancer Treatment. Adv Sci. 2019;6(10):1802157. doi:10.1002/advs.201802157

53. Verfaillie T, Garg AD, Agostinis P. Targeting ER stress induced apoptosis and inflammation in cancer. Cancer Lett. 2013;332 (2):249-264. doi:10.1016/j.canlet.2010.07.016

54. Li W, Yang J, Luo L, et al. Targeting photodynamic and photothermal therapy to the endoplasmic reticulum enhances immunogenic cancer cell death. Nat Commun. 2019;10(1):3349. doi:10.1038/s41467-019-11269-8

55. Ni J, Bucci J, Chang L, Malouf D, Graham P, Li Y. Targeting MicroRNAs in Prostate Cancer Radiotherapy. Theranostics. 2017;7(13):3243-3259. doi:10.7150/thno.19934
56. Golden EB, Pellicciotta I, Demaria S, Barcellos-Hoff $\mathrm{MH}$, Formenti SC. The convergence of radiation and immunogenic cell death signaling pathways. Front Oncol. 2012;2:88. doi:10.3389/fonc. 2012.00088

57. Dou Y, Liu Y, Zhao F, et al. Radiation-responsive scintillating nanotheranostics for reduced hypoxic radioresistance under ROS/ NO-mediated tumor microenvironment regulation. Theranostics. 2018;8(21):5870-5889. doi:10.7150/thno.27351

58. Ghoodarzi R, Changizi V, Montazerabadi AR, Eyvazzadaeh N. Assessing of integration of ionizing radiation with Radachlorin-PDT on MCF-7 breast cancer cell treatment. Lasers Med Sci. 2016;31(2):213-219. doi:10.1007/s10103-015-1844-0

59. Nakano A, Watanabe D, Akita Y, Kawamura T, Tamada Y, Matsumoto Y. Treatment efficiency of combining photodynamic therapy and ionizing radiation for Bowen's disease. $J$ Eur Acad Dermatol Venereol. 2011;25(4):475-478. doi:10.1111/j.14683083.2010.03757.x

60. $\mathrm{Lu} \mathrm{K}$, He C, Guo $\mathrm{N}$, et al. Low-dose X-ray radiotherapy-radiodynamic therapy via nanoscale metal-organic frameworks enhances checkpoint blockade immunotherapy. Nat Biomed Eng. 2018;2(8):600-610. doi:10.1038/s41551-018-0203-4

61. Wang H, Lv B, Tang Z, et al. Scintillator-Based Nanohybrids with Sacrificial Electron Prodrug for Enhanced X-ray-Induced Photodynamic Therapy. Nano Lett. 2018;18(9):5768-5774. doi:10.1021/acs.nanolett.8b02409

62. Sun W, Luo L, Feng Y, et al. Aggregation-Induced Emission Gold Clustoluminogens for Enhanced Low-Dose X-ray-Induced Photodynamic Therapy. Angew Chem Int Ed Engl. 2020;59 (25):9914-9921. doi:10.1002/anie.201908712

63. Doix B, Trempolec N, Riant O, Feron O. Low Photosensitizer Dose and Early Radiotherapy Enhance Antitumor Immune Response of Photodynamic Therapy-Based Dendritic Cell Vaccination. Front Oncol. 2019;9:811. doi:10.3389/ fonc. 2019.00811

64. Trempolec N, Doix B, Degavre C, et al. Photodynamic Therapy-Based Dendritic Cell Vaccination Suited to Treat Peritoneal Mesothelioma. Cancers. 2020;12:3. doi:10.3390/ cancers 12030545

65. Wang Z, Cao YJ. Adoptive Cell Therapy Targeting Neoantigens: a Frontier for Cancer Research. Front Immunol. 2020;11:176. doi:10.3389/fimmu.2020.00176

66. Jiang X, Xu J, Liu M, et al. Adoptive CD8(+) T cell therapy against cancer: challengesand opportunities. Cancer Lett. 2019;462:23-32. doi:10.1016/j.canlet.2019.07.017

67. Chen Q, Hu Q, Dukhovlinova E, et al. Photothermal Therapy Promotes Tumor Infiltration and Antitumor Activity of CAR T Cells. Adv Mater. 2019;31(23):e1900192. doi:10.1002/ adma.201900192

68. Korbelik M, Sun J. Cancer treatment by photodynamic therapy combined with adoptive immunotherapy using genetically altered natural killer cell line. Int $J$ Cancer. 2001;93(2):269-274. doi:10.1002/ijc.1326

69. Fang L, Zhao Z, Wang J, et al. Engineering autologous tumor cell vaccine to locally mobilize antitumor immunity in tumor surgical bed. Sci Adv. 2020;6(25):eaba4024. doi:10.1126/sciadv.aba4024

70. Son S, Kim JH, Wang X, et al. Multifunctional sonosensitizers in sonodynamic cancer therapy. Chem Soc Rev. 2020;49 (11):3244-3261. doi:10.1039/C9CS00648F

71. Xu M, Zhou L, Zheng L, et al. Sonodynamic therapy-derived multimodal synergistic cancer therapy. Cancer Lett. 2021;497:229-242. doi:10.1016/j.canlet.2020.10.037

72. Liang S, Deng X. Recent Advances in Nanomaterial-Assisted Combinational Sonodynamic Cancer Therapy. Adv Mater. 2020;32(47):2003214. doi:10.1002/adma.202003214 
73. Zheng L, Zhang $\mathrm{Y}$, Lin $\mathrm{H}$, et al. Ultrasound and Near-Infrared Light Dual-Triggered Upconversion Zeolite-Based Nanocomposite for Hyperthermia-Enhanced Multimodal Melanoma Therapy via a Precise Apoptotic Mechanism. ACS Appl Mater Interfaces. 2020;12(29):32420-32431. doi:10.1021/acsami.0c07297

74. Liu Z, Wang D, Li J, Jiang Y. Self-assembled peptido-nanomicelles as an engineered formulation for synergy-enhanced combinational SDT, PDT and chemotherapy to nasopharyngeal carcinoma. Chem Commun (Camb). 2019;55 (69):10226-10229. doi:10.1039/C9CC05463D

75. Wan Z, Sun R, Moharil P, et al. Research advances in nanomedicine, immunotherapy, and combination therapy for leukemia. $J$ Leukoc Biol. 2021;109(2):425-436. doi:10.1002/jlb.5mr0620-063rr

76. Cramer GM, Moon EK, Cengel KA, Busch TM. Photodynamic Therapy and Immune Checkpoint Blockade†. Photochem Photobiol. 2020;96(5):954-961. doi:10.1111/php.13300

77. $\mathrm{Li} \mathrm{M}$, Bolduc $\mathrm{AR}$, Hoda $\mathrm{MN}$, et al. The indoleamine 2,3-dioxygenase pathway controls complement-dependent enhancement of chemo-radiation therapy against murine glioblastoma. J Immunother Cancer. 2014;2:21. doi:10.1186/ 2051-1426-2-21

78. Schadendorf D, Hodi FS, Robert C, et al. Pooled Analysis of Long-Term Survival Data From Phase II and Phase III Trials of Ipilimumab in Unresectable or Metastatic Melanoma. $J$ Clin Oncol. 2015;33(17):1889-1894. doi:10.1200/jco.2014.56.2736

79. Garon EB, Rizvi NA, Hui R, et al. Pembrolizumab for the treatment of non-small-cell lung cancer. $N$ Engl J Med. 2015;372 (21):2018-2028. doi:10.1056/NEJMoa1501824

80. Zou W. Immunosuppressive networks in the tumour environment and their therapeutic relevance. Nat Rev Cancer. 2005;5 (4):263-274. doi:10.1038/nrc1586

81. Munn DH, Zhou M, Attwood JT, et al. Prevention of allogeneic fetal rejection by tryptophan catabolism. Science. 1998;281 (5380):1191-1193. doi:10.1126/science.281.5380.1191

82. Yue EW, Douty B, Wayland B, et al. Discovery of potent competitive inhibitors of indoleamine 2,3-dioxygenase with in vivo pharmacodynamic activity and efficacy in a mouse melanoma model. J Med Chem. 2009;52(23):7364-7367. doi:10.1021/ jm900518f

83. Nayak-Kapoor A, Hao Z, Sadek R, et al. Phase Ia study of the indoleamine 2,3-dioxygenase 1 (IDO1) inhibitor navoximod (GDC-0919) in patients with recurrent advanced solid tumors. $J$ Immunother Cancer. 2018;6(1):61. doi:10.1186/s40425-0180351-9

84. $\mathrm{Lu} \mathrm{K}, \mathrm{He} \mathrm{C}$, Guo N, et al. Chlorin-Based Nanoscale Metal-Organic Framework Systemically Rejects Colorectal Cancers via Synergistic Photodynamic Therapy and Checkpoint Blockade Immunotherapy. $J$ Am Chem Soc. 2016;138 (38):12502-12510. doi:10.1021/jacs.6b06663

85. Liu D, Chen B, Mo Y, et al. Redox-Activated Porphyrin-Based Liposome Remote-Loaded with Indoleamine 2,3-Dioxygenase (IDO) Inhibitor for Synergistic Photoimmunotherapy through Induction of Immunogenic Cell Death and Blockage of IDO Pathway. Nano Lett. 2019;19(10):6964-6976. doi:10.1021/acs. nanolett.9b02306

86. Zhao LP, Zheng RR, Huang JQ, et al. Self-Delivery Photo-Immune Stimulators for Photodynamic Sensitized Tumor Immunotherapy. ACS Nano. 2020. doi:10.1021/acsnano.0c06765

87. Wing K, Onishi Y, Prieto-Martin P, et al. CTLA-4 control over Foxp3+ regulatory $\mathrm{T}$ cell function. Science. 2008;322 (5899):271-275. doi:10.1126/science.1160062

88. Xu J, Xu L, Wang C, et al. Near-infrared-triggered photodynamic therapy with multitasking upconversion nanoparticles in combination with checkpoint blockade for immunotherapy of colorectal cancer. ACS Nano. 2017;11(5):4463-4474. doi:10.1021/ acsnano.7b00715
89. Lin B, Liu J, Wang Y, Yang F, Huang L, Lv R. Enhanced Upconversion Luminescence-Guided Synergistic Antitumor Therapy Based on Photodynamic Therapy and Immune Checkpoint Blockade. Chem Mater. 2020;32(11):4627-4640. doi:10.1021/acs.chemmater.0c01031

90. Wang Z, Zhang F, Shao D, et al. Janus Nanobullets Combine Photodynamic Therapy and Magnetic Hyperthermia to Potentiate Synergetic Anti-Metastatic Immunotherapy. Adv Sci. 2019;6 (22): 1901690. doi:10.1002/advs.201901690

91. Zou W, Wolchok JD, Chen L. PD-L1 (B7-H1) and PD-1 pathway blockade for cancer therapy: mechanisms, response biomarkers, and combinations. Sci Transl Med. 2016;8(328):328rv324. doi:10.1126/scitranslmed.aad7118

92. Dong H, Strome SE, Salomao DR, et al. Tumor-associated B7-H1 promotes T-cell apoptosis: a potential mechanism of immune evasion. Nat Med. 2002;8(8):793-800. doi:10.1038/nm730

93. Duan X, Chan C, Guo N, Han W, Weichselbaum RR, Lin W. Photodynamic therapy mediated by nontoxic core-shell nanoparticles synergizes with immune checkpoint blockade to elicit antitumor immunity and antimetastatic effect on breast cancer. $J$ Am Chem Soc. 2016;138(51):16686-16695. doi:10.1021/jacs.6 b09538

94. Zhang R, Zhu Z, Lv H, et al. Immune Checkpoint Blockade Mediated by a Small-Molecule Nanoinhibitor Targeting the PD-1/PD-L1 Pathway Synergizes with Photodynamic Therapy to Elicit Antitumor Immunity and Antimetastatic Effects on Breast Cancer. Small. 2019;15(49):e1903881. doi:10.1002/ smll.201903881

95. Li M, Shao Y, Kim JH, et al. Unimolecular Photodynamic $\mathrm{o}_{2}$ -economizer to overcome hypoxia resistance in phototherapeutics. $J$ Am Chem Soc. 2020;142(11):5380-5388. doi:10.1021/ jacs.0c00734

96. Sun F, Zhu Q, Li T, et al. Regulating Glucose Metabolism with Prodrug Nanoparticles for Promoting Photoimmunotherapy of Pancreatic Cancer. Adv Sci. 2021;8(4):2002746. doi:10.1002/ advs.202002746

97. Xiong W, Qi L, Jiang N, et al. Metformin Liposome-Mediated PD-L1 Downregulation for Amplifying the Photodynamic Immunotherapy Efficacy. ACS Appl Mater Interfaces. 2021;13 (7):8026-8041. doi:10.1021/acsami.0c21743

98. Cha JH, Yang WH, Xia W, et al. Metformin Promotes antitumor immunity via endoplasmic-reticulum-associated degradation of PD-L1. Mol Cell. 2018;71(4):606-620.e607. doi:10.1016/j. molcel.2018.07.030

99. Ruby CE, Montler R, Zheng R, Shu S, Weinberg AD. IL-12 is required for anti-OX40-mediated CD4 T cell survival. J Immunol. 2008;180(4):2140-2148. doi:10.4049/jimmunol.180.4.2140

100. Valzasina B, Guiducci C, Dislich H, Killeen N, Weinberg AD, Colombo MP. Triggering of OX40 (CD134) on CD4(+)CD25+ $\mathrm{T}$ cells blocks their inhibitory activity: a novel regulatory role for OX40 and its comparison with GITR. Blood. 2005;105 (7):2845-2851. doi:10.1182/blood-2004-07-2959

101. Kroemer A, Xiao X, Vu MD, et al. OX40 controls functionally different $\mathrm{T}$ cell subsets and their resistance to depletion therapy. J Immunol. 2007;179(8):5584-5591. doi:10.4049/jimmunol.17 9.8.5584

102. Kuang Z, Jing H, Wu Z, et al. Development and characterization of a novel anti-OX40 antibody for potent immune activation. Cancer Immunol Immunother. 2020;69(6):939-950. doi:10.1007/ s00262-020-02501-2

103. Gough MJ, Crittenden MR, Sarff M, et al. Adjuvant therapy with agonistic antibodies to CD134 (OX40) increases local control after surgical or radiation therapy of cancer in mice. $J$ Immunother. 2010;33(8):798-809. doi:10.1097/CJI.0b013 e3181ee7095 
104. Yokouchi H, Yamazaki K, Chamoto K, et al. Anti-OX40 monoclonal antibody therapy in combination with radiotherapy results in therapeutic antitumor immunity to murine lung cancer. Cancer Sci. 2008;99(2):361-367. doi:10.1111/j.1349-7006.2007.00664.x

105. Hirschhorn-Cymerman D, Rizzuto GA, Merghoub T, et al. OX40 engagement and chemotherapy combination provides potent antitumor immunity with concomitant regulatory $\mathrm{T}$ cell apoptosis. J Exp Med. 2009;206(5):1103-1116. doi:10.1084/jem.20082205

106. Alvim R, Nogueira L, Georgala P, et al. Combined OX40 agonist and PD-1 inhibitor immunotherapy improves the efficacy of vascular targeted photodynamic therapy in a urothelial tumor model. $J$ Clin Oncol. 2020;38:e17004-e17004. doi:10.1200/ JCO.2020.38.15_suppl.e17004

107. Anderson AC, Joller N, Kuchroo VK. Lag-3, Tim-3, and TIGIT: co-inhibitory Receptors with Specialized Functions in Immune Regulation. Immunity. 2016;44(5):989-1004. doi:10.1016/j. immuni.2016.05.001

108. de Mingo Pulido A, Gardner A, Hiebler S, et al. TIM-3 Regulates CD103(+) Dendritic Cell Function and Response to Chemotherapy in Breast Cancer. Cancer Cell. 2018;33(1):6074.e66. doi:10.1016/j.ccell.2017.11.019

109. Kim JE, Patel MA, Mangraviti A, et al. Combination Therapy with Anti-PD-1, Anti-TIM-3, and Focal Radiation Results in Regression of Murine Gliomas. Clin Cancer Res. 2017;23 (1):124-136. doi:10.1158/1078-0432.ccr-15-1535

110. Anzengruber F, Avci P, de Freitas LF, Hamblin MR. T-cell mediated anti-tumor immunity after photodynamic therapy: why does it not always work and how can we improve it? Photochem Photobiol Sci. 2015;14(8):1492-1509. doi:10.1039/c4pp00455h

111. Ng C, Jingchao L, Pu K. Recent progresses in phototherapy-synergized cancer immunotherapy. Adv Funct Mater. 2018;28:1804688. doi:10.1002/adfm.201804688

112. Wang J, Gao ZP, Qin S, Liu CB, Zou LL. Calreticulin is an effective immunologic adjuvant to tumor-associated antigens. Exp Ther Med. 2017;14(4):3399-3406. doi:10.3892/ etm.2017.4989

113. Xia Y, Gupta GK, Castano AP, Mroz P, Avci P, Hamblin MR. $\mathrm{CpG}$ oligodeoxynucleotide as immune adjuvant enhances photodynamic therapy response in murine metastatic breast cancer. J Biophotonics. 2014;7(11-12):897-905. doi:10.1002/ jbio. 201300072

114. Chen WR, Korbelik M, Bartels KE, Liu H, Sun J, Nordquist RE. Enhancement of laser cancer treatment by a chitosan-derived immunoadjuvant. Photochem Photobiol. 2005;81(1):190-195. doi:10.1562/2004-07-20-ra-236

115. Korbelik M, Banáth J, Zhang W, et al. N-dihydrogalactochitosan as immune and direct antitumor agent amplifying the effects of photodynamic therapy and photodynamic therapy-generated vaccines. Int Immunopharmacol. 2019;75:105764. doi:10.1016/j. intimp.2019.105764
116. Hwang HS, Cherukula K, Bang YJ, et al. Combination of Photodynamic Therapy and a Flagellin-Adjuvanted Cancer Vaccine Potentiated the Anti-PD-1-Mediated Melanoma Suppression. Cells. 2020;9(11). doi:10.3390/cells9112432

117. Ni K, Luo T, Culbert A, Kaufmann M, Jiang X, Lin W. Nanoscale Metal-Organic Framework Co-delivers TLR-7 Agonists and Anti-CD47 Antibodies to Modulate Macrophages and Orchestrate Cancer Immunotherapy. J Am Chem Soc. 2020;142 (29):12579-12584. doi:10.1021/jacs.0c05039

118. Korbelik M, Cecic I. Enhancement of tumour response to photodynamic therapy by adjuvant mycobacterium cell-wall treatment. J Photochem Photobiol B. 1998;44(2):151-158. doi:10.1016/ s1011-1344(98)00138-9

119. Wculek SK, Cueto FJ, Mujal AM, Melero I, Krummel MF, Sancho D. Dendritic cells in cancer immunology and immunotherapy. Nat Rev Immunol. 2020;20(1):7-24. doi:10.1038/s41577-019-0210-z

120. Jung NC, Kim HJ, Kang MS, et al. Photodynamic therapy-mediated DC immunotherapy is highly effective for the inhibition of established solid tumors. Cancer Lett. 2012;324 (1):58-65. doi:10.1016/j.canlet.2012.04.024

121. Zheng Y, Yin G, Le V, et al. Photodynamic-therapy Activates Immune Response by disrupting Immunity Homeostasis of Tumor Cells, which Generates Vaccine for Cancer Therapy. Int J Biol Sci. 2016;12(1):120-132. doi:10.7150/ijbs.12852

122. Zhang H, Wang P, Wang X, et al. Antitumor Effects of DC Vaccine With ALA-PDT-Induced immunogenic apoptotic cells for skin squamous cell carcinoma in mice. Technol Cancer Res Treat. 2018;17:1533033818785275. doi:10.1177/ 1533033818785275

123. Kleinovink JW, van Driel PB, Snoeks TJ, et al. Combination of photodynamic therapy and specific immunotherapy efficiently eradicates established tumors. Clin Cancer Res. 2016;22 (6):1459-1468. doi:10.1158/1078-0432.ccr-15-0515

124. Zheng L, Li Y, Cui Y, et al. Generation of an effective anti-lung cancer vaccine by DTPP-mediated photodynamic therapy and mechanistic studies. Lasers Med Sci. 2013;28(5):1383-1392. doi:10.1007/s10103-013-1270-0

125. Liu X, Cheng JC, Turner LS, et al. Acid ceramidase upregulation in prostate cancer: role in tumor development and implications for therapy. Expert Opin Ther Targets. 2009;13(12):1449-1458. doi:10.1517/14728220903357512

126. Korbelik M, Banáth J, Zhang W, et al. Interaction of acid ceramidase inhibitor LCL521 with tumor response to photodynamic therapy and photodynamic therapy-generated vaccine. Int J Cancer. 2016;139(6):1372-1378. doi:10.1002/ijc.30171
International Journal of Nanomedicine

\section{Publish your work in this journal}

The International Journal of Nanomedicine is an international, peerreviewed journal focusing on the application of nanotechnology in diagnostics, therapeutics, and drug delivery systems throughout the biomedical field. This journal is indexed on PubMed Central, MedLine, CAS, SciSearch ${ }^{\mathbb{}}$, Current Contents ${ }^{\mathbb{R}} /$ Clinical Medicine,
Journal Citation Reports/Science Edition, EMBase, Scopus and the Elsevier Bibliographic databases. The manuscript management system is completely online and includes a very quick and fair peer-review system, which is all easy to use. Visit http://www.dovepress.com/ testimonials.php to read real quotes from published authors. 